\title{
过渡金属催化醇与胺有氧脱水反应及相关研究进展
}

\author{
徐 清* 李 强 \\ (温州大学化学与材料工程学院 温州 325035)
}

\begin{abstract}
摘要 与其他胺和酰胺衍生物的合成方法相比, 过渡金属催化醇与各类胺和酰胺的脱水 $N$-烷基化反应是一种相对绿 色、原子经济性较高的方法，一般被称为 “借氢” 或 “氢自动转移” 反应及其方法学. 近年来，在空气氛围下过渡金属 催化醇与胺和酰胺的有氧脱水 $N$-烷基化反应，可使用更稳定的金属催化剂、可在无配体、空气等更温和简单的条件下 进行，也引起了人们的极大关注. 主要介绍近年来过渡金属催化下醇与胺和酰胺在空气或者氧化剂作用下构建 $\mathrm{C}-\mathrm{N}$, $\mathrm{C}=\mathrm{N}$ 键合成胺和酰胺衍生物以及亚胺类化合物的有氧脱水反应进展情况，同时也对相关有氧脱水 $C$-烷基化反应进行 简单介绍. 相关反应的机理研究也将作适当讨论.
\end{abstract}

关键词 过渡金属催化; 醇; 胺和酰胺衍生物; $N$-烷基化反应; 亚胺化反应; 有氧氧化反应; 接力方法学

\section{Recent Advances of Transition Metal-Catalyzed Aerobic Dehydrative Reactions of Alcohols and Amines and Related Researches}

\author{
Xu, Qing* Li, Qiang \\ (College of Chemistry and Materials Engineering, Wenzhou University, Wenzhou 325035)
}

\begin{abstract}
In comparison with other methods, transition metal-catalyzed dehydrative $N$-alkylation of amines and amides with alcohols, commonly known as the borrowing hydrogen or hydrogen autotransfer reactions and the methodology, is a comparatively green and atom-economic method for the synthesis of the useful amine and amide derivatives. Recently, transition metal-catalyzed aerobic dehydrative $\mathrm{N}$-alkylation method has also attracted much attention, for the reactions can be readily conducted under milder and simpler conditions by using the more stable metal catalysts under the air atmosphere. In this review, we summarize the recent advances of transition metal-catalyzed aerobic dehydrative $\mathrm{C}-\mathrm{N}$ and $\mathrm{C}=\mathrm{N}$ bond forming reactions of alcohols with amines and amides for the synthesis of the amine and amide derivatives and imines, as well as those of the related aerobic dehydrative $C$-alkylation reactions. Mechanisms of the above reactions are also discussed.
\end{abstract}

Keywords transition metal catalysis; alcohols; amine and amide derivatives; $N$-alkylation reaction; imination reaction; aerobic oxidative reaction; relay race methodology

氮是生命必需元素，因此有机氮化合物如各种胺、 酰胺、亚胺及其衍生物是有机和药物化学中非常重要的 一类氮源和合成中间体 ${ }^{[1,2]}$. 各种胺和酰胺结构不仅广 泛存在于具有生理和药物活性的化合物和天然产物中, 也是构成一些生物体和生命活动不可缺少的物质 ${ }^{[1]}$. 而 含活泼 $\mathrm{C}=\mathrm{N}$ 不饱和双键的亚胺类化合物, 由于可发生 氢化、加成、缩合、环加成、多组分等各种类型的反应 来构建 $\mathrm{C}-\mathrm{C}$ 和 $\mathrm{C}-\mathrm{E}$ (杂原子)键, 在有机、药物、天然 产物和工业合成中都具有极其重要的应用 ${ }^{[2]}$. 因此, 这
些有机氮化合物的合成和应用研究一直都是有机化学 研究的中心内容之一. 随着人们环境保护意识的不断增 强，开发高原子经济性、更加绿色的、催化进行的新方 法以替代原有的步骤长、污染大、缺点多的传统方法来 合成这些胺、酰胺和亚胺衍生物, 不仅是有机合成和金 属有机化学研究发展的趋势, 对于制药和工业合成研究 领域而言也具有重要的指导意义.

在金属有机化学迅速发展的大背景下，过渡金属催 化的方法越来越多的被运用到胺和酰胺衍生物的合成

*E-mail: qing-xu@wzu.edu.cn

Received August 17, 2012; revised September 22, 2012; published online October 8, 2012.

Project supported by the Startup Funding of Wenzhou University, the National Natural Science Foundation of China (No. 20902070), the Scientific Research Foundation for the Returned Overseas Chinese Scholars of State Education Ministry, the Natural Science Foundation of Zhejiang Province (No. Y4100579) and the Qianjiang Talents Program of Zhejiang Province (No. QJD0902004).

温州大学引进人才科研启动经费、国家自然科学基金(No. 20902070)、教育部第 40 批留学回国人员科研启动金、浙江省自然科学基金(No. Y4100579) 和钱江人才计划 D 类(No. QJD0902004)资助项目. 
中，例如卤代芳烃与 $\mathrm{N}-\mathrm{H}$ 键的 Buchwald-Hartwig 胺化 反应 ${ }^{[3]}$ 、羰基化合物与胺在氢气或者还原剂存在下的还 原胺化反应 ${ }^{[4]} 、 \mathrm{~N}-\mathrm{H}$ 键对 $\mathrm{C}-\mathrm{C}$ 不饱和键的加成反应 ${ }^{[5]}$, 以及其他类型的反应 ${ }^{[6,7]}$. 胺与酰胺类化合物的 $N$-烷基 化反应研究方面, 如 Scheme 1 所示, 主要有(a)传统的胺 与卤代烃的 Hoffmann $N$-烷基化反应 ${ }^{[7]}$ 和(b)亚胺的还原 反应 ${ }^{[2]}$ 及相关的羰基化合物的还原胺化反应 ${ }^{[4]}$. 但是这 两种方法缺点也很明显, 需使用活性较高、毒性较大的 卤代烃或者醛酮, 还产生大量的副产物、废弃物, Hoffmann $N$-烷基化反应还得到各种胺产物和季铵盐的 混合物，选择性低、难于分离.

由于很多卤代烃和醛酮化合物一般可由相应的醇 $\mathbf{1}$ 经卤化或者氧化反应制备, 如果可以直接使用醇为反应 原料与胺 2 进行脱水的 $N$-烷基化反应合成相应的胺衍 生物 3 (Scheme 1, 式 c), 不仅可以使反应在较低条件下 进行、减少反应步骤、减少消耗和污染, 唯一的副产物 为水, 而且可以使用低价易得、稳定低毒、来源更广的 醇类为烷基化试剂. 因此, 醇与胺的脱水 $N$-烷基化反应 是对 Hoffmann 反应 ${ }^{[7]}$ 和亚胺还原反应等多步 $N$-烷基化 方法的极大改进, 也是一种原子经济性较高、更加绿色、 对环境也更加友好的方法.

实际上, 醇与胺脱水的 $N$-烷基化反应是一个比较 古老的反应. 1901 年, $\mathrm{Nef}^{[8]}$ 报道了首例醇与胺合成胺衍 生物的反应(Scheme 2). 该方法使用乙醇钠与苯胺或乙 酰苯胺反应, 在 $250 \sim 305{ }^{\circ} \mathrm{C}$ 的高温下可分别得到 $20 \%$ 和 $40 \%$ 的收率的产物.

此后, 其他研究者也相继报道了不少烷氧基铝等与 胺、或者醇与胺在强碱的作用下的脱水 $N$-烷基化反应 ${ }^{[9]}$, 对 Nef 的方法进行改进. 由于醇的活性相对较低, 这些 方法往往需要很苛刻的反应条件, 如高温(一般 $>200$ ${ }^{\circ} \mathrm{C}$, 甚至高达 $400{ }^{\circ} \mathrm{C}$ )、高压、使用过量的醇或胺、使 用过量碱的强碱性条件, 而且有些反应时间长、收率低、 选择性低. 受到苛刻的反应条件的制约, 早期醇与胺的 $N$-烷基化反应研究总体上进展不大 ${ }^{[9]}$.
1981 1982 年, Grigg, Watanabe, Murahashi 等小 组 ${ }^{[10]}$ 相继独立报道了最早的均相过渡金属催化剂催化 的醇与胺的脱水 $N$-烷基化反应. 此后, 基于过渡金属催 化的醇的活化及醇与胺的脱水 $N$-烷基化反应研究受到 人们的普遍重视, 并被逐渐扩展到烷烃和胺的活化、非 均相过渡金属催化剂催化反应、以及脱水 $\mathrm{C}-\mathrm{C}$ 键的构 建等研究中 ${ }^{[9,11]}$, 成为当前有机合成、金属有机催化反 应和绿色化学等领域相对热门的研究方向.

采用过渡金属催化剂可以对不活泼的醇羟基进行 有效的活化, 使得反应可在更低的温度和更温和的条件 下进行, 产物的选择性和产率也更高. 目前已有不少综 述总结了这些反应 ${ }^{[9,11]}$. 如 Scheme 3 所示, 一般认为, 这些过渡金属催化的醇与胺的脱水 $N$-烷基化反应经过 如下的机理进行: 首先(步骤 $\mathrm{i}$ ), 金属催化剂 $[\mathrm{M}]$ 从原料 醇 1 中 “借得” 氢, 形成 $[\mathrm{MH}]$ 或 $\left[\mathrm{MH}_{2}\right]$, 同时将醇转化 为较活泼的醛 4 , 这一过程也称为醇的活化; 接着(步骤 ii), 醛与胺 $\mathbf{2}$ 脱水缩合生成亚胺中间体 $\mathbf{5}$; 最后(步骤 iii), 亚胺 5 被 $[\mathrm{MH}]$ 或 $\left[\mathrm{MH}_{2}\right]$ 还原, 即 $[\mathrm{MH}]$ 或 $\left[\mathrm{MH}_{2}\right]$ 将借来的 氢 “还给” 亚胺, 生成饱和的目标胺产物 $\mathbf{3}$, 并再生催化 剂[M]. 对此, 英国的 Williams 形象地称之为 “借氢” (borrowing Hydrogen)机理 ${ }^{[1 \mathrm{a} \sim \mathrm{c}]}$, 西班牙的 Ramón 和 Yus 提出 “氢自动转移” 机理(hydrogen autotransfer)的 说法 ${ }^{[9,11 \mathrm{~h}]}$, 而日本的 Fujita 和 Yamaguchi 则将其简单地 称为 “氢转移” 方法(hydrogen transfer process) ${ }^{[11 i]}$. 三种 提法, 究其本质是一样的, 都认为反应经过形成金属氢 化物 $[\mathrm{MH}]$ 或 $\left[\mathrm{MH}_{2}\right]$ 的机理进行 (Scheme 3). 这一类利用 醇为脱水烷基化试剂的反应和方法也被 Williams 和 Yus 等称为 “借氢” 或 “氢自动转移” 方法学.

借氢反应一般使用贵重过渡金属 $R u$ (钓)或 $\operatorname{Ir}$ (铱)的 络合为催化剂, 在弱碱性或强碱的条件下进行, 主要适 用于伯醇和伯胺的反应，可用于高选择地合成二级胺. 相比之下, 仲醇和仲胺的反应相对较少. 仲胺由于难以 脱水形成亚胺中间体, 理论上较难发生反应; 而叔醇因 为自身不能脱氢, 不可能发生借氢类型的反应.

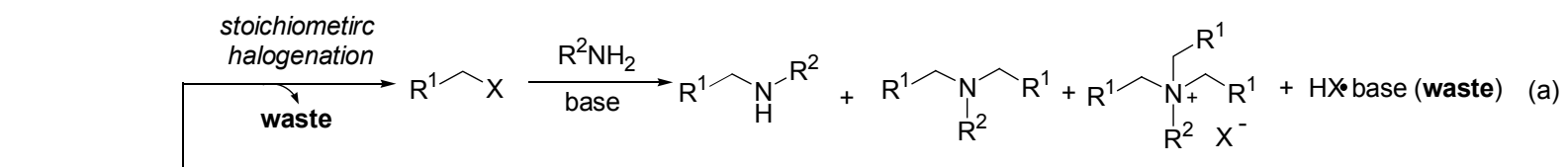

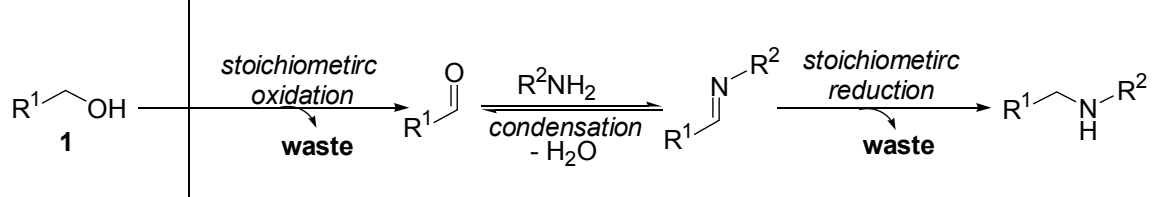

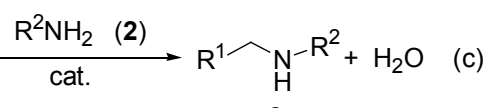

$$
\begin{aligned}
& 3
\end{aligned}
$$

Scheme 1 


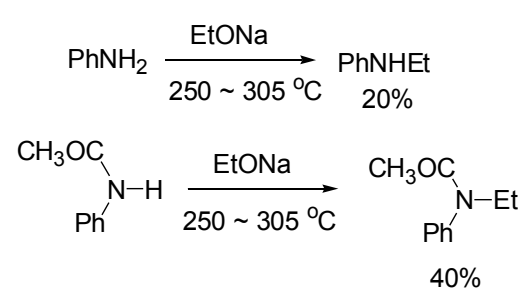

Scheme 2

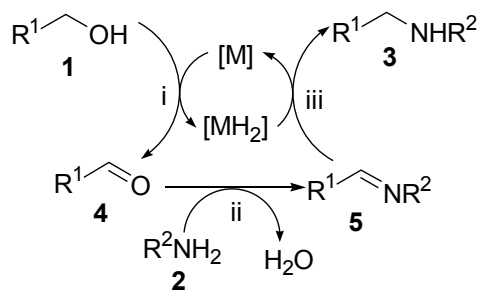

Scheme 3

然而, 醇的脱氢活化和金属氢化物的形成(Scheme 3 , 步骤 i), 实际上在热力学上是一个不利的过程 ${ }^{[11 f]}$. 因 此, 典型的借氢反应需使用昂贵的 Ru, Ir 催化剂结合复 杂的配体才能体现较好的催化效果. 很多贵金属催化 剂、配体尚属实验室制备和研究阶段, 一般条件下(如空 气下)不稳定、不易大量制备, 价格也无法估算. 加上形 成的敏感活泼的 $[\mathrm{MH}]$ 或 $\left[\mathrm{MH}_{2}\right]$ 络合物容易失活，典型借 氢反应需在惰性气体保护下进行, 也导致催化剂的制备 和反应的进行往往需要使用手套箱等设备来完成. 加上 重金属的毒性和残留问题, 各种不利因素导致借氢反应 难以扩大规模, 限制了其进一步推广和应用的潜力. 虽 然非均相、负载的、可回收的过渡金属催化剂的开发和
研究也有不少报道 ${ }^{[9,11]}$, 但是其反应效果往往不如均相 催化剂. 因此, 开发稳定易得的金属催化剂、或者廉价 过渡金属的催化剂、以及温和简便的新方法，实现高效 高选择的醇与胺和酰胺化合物的脱水 $N$-烷基化反应，以 弥补借氢方法的弊端和改进脱水 $N$-烷基化方法，非常值 得研究.

此外, 文献上报道的另一种类型的醇与胺的脱水 $N$ 烷基化反应不容忽视. 实际上，醇与胺的脱水反应受底 物、催化剂性质和反应条件的影响较大，在仲醇、烯丙 醇、炔丙醇、叔醇的情况下，相应的反应一般通过醇羟 基的直接亲核取代反应进行(Scheme 4) ${ }^{[12]}$. 这类反应近 几年也得到了较好的研究，也有一些综述对其进行了总 结 ${ }^{[2 a, 12 b]}$. 醇的直接亲核取代反应与借氢反应在很多方 面有很大的不同. 除了反应底物不同外，它们一般使用 路易斯酸或者布朗酸、或者一些过渡金属络合物(根据定 义，很多过渡金属催化剂实际上也属于路易斯 酸 ${ }^{[12 \mathrm{k} \sim 120]}$ 为催化剂, 在酸性(或无碱)条件下, 反应非常 容易通过形成稳定的碳正离子中间体或金属络合的碳 正离子中间体的机理进行. 研究显示, 简单的伯醇在某 些情况下也能发生这种类型的反应 ${ }^{[12 g ~ 12 j]}$.

最新报道显示，一些过渡金属催化的醇 1 与胺和酰 胺 2 、仲醇 $\mathbf{6}$ 或甲基酮 7 的脱水 $N$-和 $C$-烷基化反应可在 空气下进行 (Scheme 5, a 和 c), 空气甚至起到了大大促 进反应的作用. 与常规惰性氛围下借氢类型的烷基化反 应 $^{[9,11]}$ 相比, 有氧烷基化反应不需要惰性气体保护, 在 反应条件和操作上更加温和、简便和实用，催化剂的选 择范围更广，还可以使用廉价和无配体的过渡金属催化

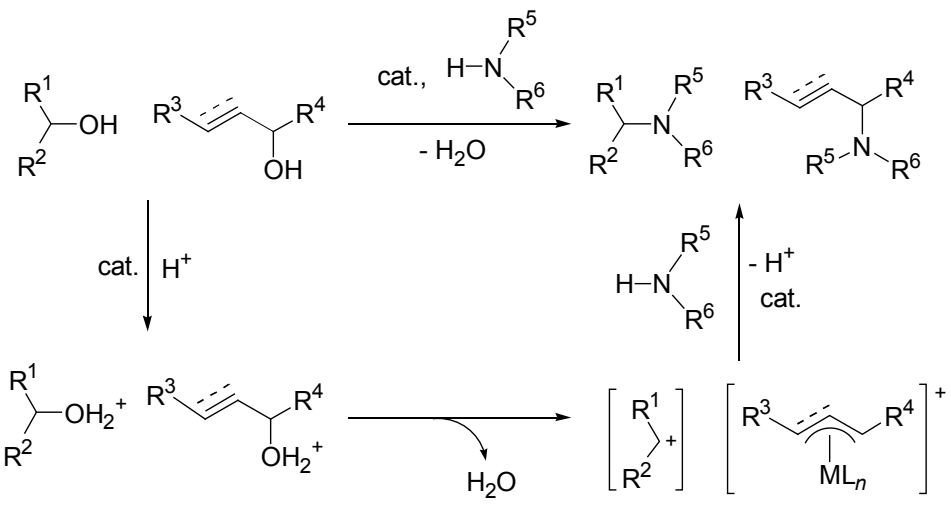

Scheme 4

(a)

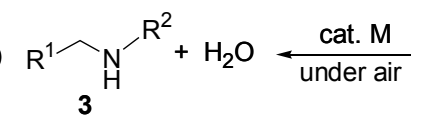

(c)

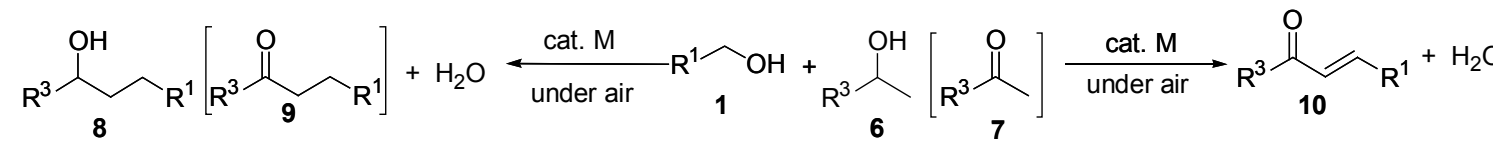

Scheme 5 
剂. 通过催化剂的篎选和反应条件的控制和优化, 还可 以将反应扩展为醇与胺或酮的有氧氧化缩合反应应用 于亚胺 5 和 $\alpha, \beta$-不饱和酮 10 等有用的含活泼 $\mathrm{C}=\mathrm{N}$ 和 $\mathrm{C}=\mathrm{C}$ 键化合物的合成 (Scheme $5, \mathrm{~b}$ 和 $\mathrm{d}$ ). 大概由于这些 在空气下进行的有氧 $N$-和 $C$-烷基化反应是新出现的一 类反应, 目前也缺乏对其进行有效的总结和研究对比, 尽管有氧烷基化反应在反应条件和反应现象上与常规 惰性氛围下典型的借氢反应存在明显区别, 不同的研究 者对反应的机理仍抱有不同的观点, 某些反应的机理研 究还存在不少争议.

因此, 本综述将主要对醇与胺和酰胺的有氧 $N$-烷基 化反应及相应的亚胺的有氧氧化缩合方法(Scheme 5, a 和 b) 进行小结, 并简单地介绍相关有氧 $C$-烷基化反应和 $\alpha, \beta$-不饱和酮的氧化缩合方法(Scheme $5, \mathrm{c}$ 和 d). 以上研 究中相关的反应机理研究也将作必要的讨论. 这些实用 的、相对绿色的 $\mathrm{C}-\mathrm{N}, \mathrm{C}-\mathrm{C}$ 键的形成反应具备不少绿 色化学的特点, 应具有良好的应用潜力、较高的应用价 值和广阔的应用前景. 同时, 目前报道的方法还存在很 大的改进空间，值得进一步的研究、开发和改进.

\section{1 醇与胺和酰胺的有氧 $N$-烷基化反应}

\section{1 醇与胺的多步 $N$-烷基化反应}

醇与胺的 $N$-烷基化反应中, 有一类反应通过分步加 入当量氧化剂和还原剂的方式实现烷基化的胺产物的 合成. 如 Scheme 6 所示, 在该类反应中, 醇 1 首先被氧 化剂氧化为醛或酮 $\mathbf{4}$, 不经分离, 在一锅条件下加入胺 后可得到亚胺中间体 $\mathbf{5}$, 再加入过量的还原剂可将亚胺 还原成饱和胺产物 3. 这类反应直接使用醇为原料, 且 反应在一锅条件下进行, 避免了中间产物醛、酮和亚胺 化合物的分离、纯化、保存等方面的问题, 是对亚胺的 还原反应 ${ }^{[2]}$ 和还原胺化反应 ${ }^{[4]}$ (Scheme 1, 式 b) 的改进, 因此也可以称为醇与胺的多步 $N$-烷基化反应. 由于在反 应中使用了氧化剂, 这类反应也可以认为是一种有氧的 $N$-烷基化反应.

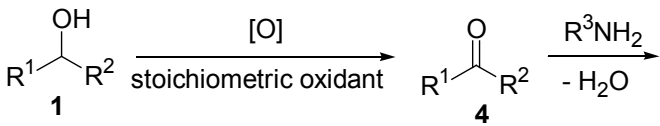

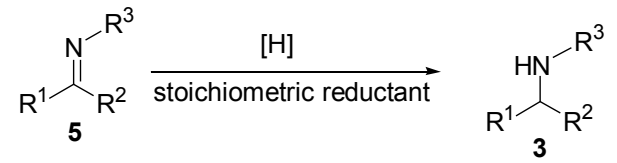

\section{Scheme 6}

2001 年, Taylor 等 ${ }^{[13]}$ 报道, 使用过量 $\mathrm{MnO}_{2}$ 为氧化 剂、高聚物负载的氰基硼氢化物(PSCBH)为还原剂, 在 $4 \AA$ 分子篮存在下, 各种苄醇与脂肪胺可经历一锅多步
的反应得到烷基化的胺产物(Eq. 1). 该反应可在较低的 温度下进行, 脂肪族的伯胺和仲胺都可以参与反应, 但 是反应时间较长, 有些反应收率比较低. 由于使用了大 大过量的氧化剂、还原剂以及一定量的添加剂, 产生了 不少的废物和污染, 也给产物的分离纯化带来一定的麻 烦, 因此该方法进一步用于应用研究的潜力有限.

$$
\begin{aligned}
& \text { (1) } \mathrm{MnO}_{2} \text { (10 equiv.) }
\end{aligned}
$$

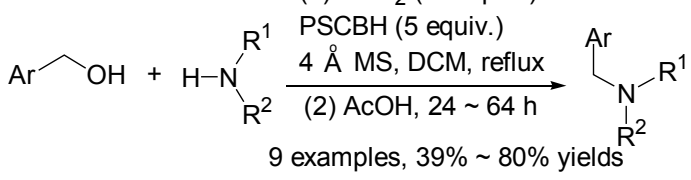

2002 年, Taylor 等 ${ }^{[14]}$ 报道了改进的方法(Eq. 2). 改 用 $\mathrm{NaBH}_{4}$ 为还原剂后, 使用 2 equiv. 即可实现较好的效 果. 新方法可将反应扩展到烯丙醇、炔丙醇、芐胺等底 物, 产物收率也有所提高. 在仲胺的反应中, 使用 $\mathrm{NaBH}_{4}$ 的效果不好，而使用 PSCBH 可得中等收率产物.
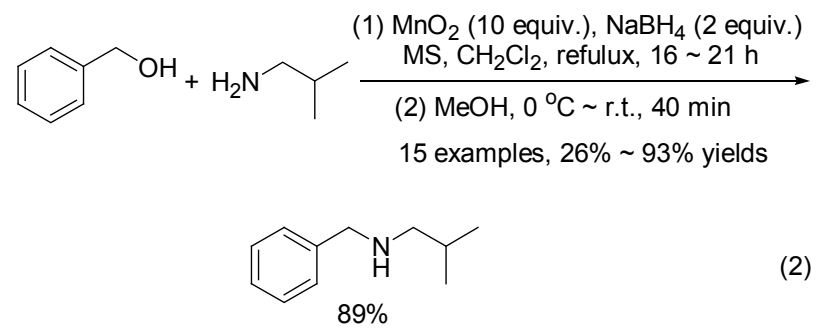

2011 年, Cossy 等 ${ }^{[15]}$ 报道了一种使用二醋酸碘苯 (BAIB)为氧化剂、TEMPO 为氧化催化剂, $\mathrm{NaBH}(\mathrm{OAc})_{3}$ 为还原剂的一锅多步的 $N$-烷基化方法(Eq. 3). 该反应 中, 醇首先被加入的 TEMPO-BAIB 体系在温和条件下 氧化为醛或酮, 然后与过量的胺缩合形成亚胺或亚胺离 子中间体，最后被 $\mathrm{NaBH}(\mathrm{OAc})_{3}$ 还原为目标 $N$-烷基化产 物. 该反应在室温下进行, 底物范围较广, 适用于一系 列脂肪醇、苠醇、仲醇、脂肪族伯胺、仲胺, 产物收率 普遍较高. 手性醇和胺也可以用于该反应, 产物的手性 没有受到影响. 虽然该方法使用的氧化剂和还原剂比 $\mathrm{MnO}_{2}$ 的方法 ${ }^{[13,14]}$ 少, 但是需使用过量的原料胺, 这在 手性胺为底物的反应中不太有利.

$$
\begin{aligned}
& \mathrm{R}^{1}, \mathrm{R}^{2}, \mathrm{R}^{3}, \mathrm{R}^{4}=\mathrm{Alkyl}, \mathrm{Aryl}, \mathrm{H} \text {, etc. } \\
& 20 \text { examples, } 60 \% \sim 96 \% \text { yields }
\end{aligned}
$$

2012 年, Cossy 等 ${ }^{[16]}$ 使用甲酸铵代替脂肪胺, 将 TEMPO-BAIB 反应体系成功地运用于伯胺的制备 
(Scheme 7). 此外, 还可用烯丙基嗍试剂替代嗍氢化物, 将还原反应扩展为加成反应，并进一步用于一些生物活 性物质的合成.

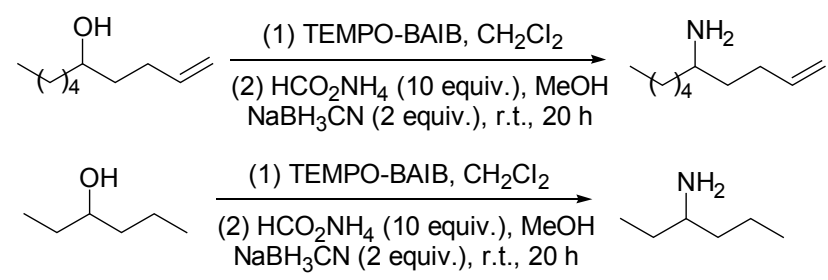

\section{Scheme 7}

\section{2 醇与胺和酰胺的有氧脱水 $N$-烷基化反应}

2009 年, Likhar 等 ${ }^{[17]}$ 报道了可在空气下进行的非均 相铜催化剂(CuAl-HT)催化的 $N$-烷基化反应(Scheme 8). 该反应需使用过量的胺和过量的碱, 并在较高的温度下 $\left(160{ }^{\circ} \mathrm{C}\right)$ 下进行。该方法适用于取代茮胺和苯胺与茮 醇、脂肪醇的反应, 其中以芐醇的反应为主. 仲胺也可 以参与反应, 得到中等到高收率的产物. 脂肪族仲醇不 反应，但二苯甲醇可得到良好收率的产物。

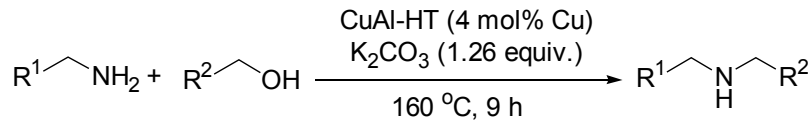

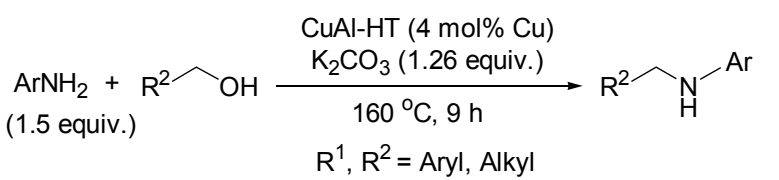

25 examples, $32 \% \sim 98 \%$ yields

\section{Scheme 8}

Likhar 等对催化剂的性质和反应机理进行了一定的 研究. 他们发现, 98\%的醛可以在无胺的空白反应中分 离得到, 因此认为反应经历醇氧化、醛胺缩合生成亚胺、 亚胺被还原为胺的过程. 由于在反应中没有检测到亚胺 中间体，以及没有观察到亚胺被催化剂(CuAl-HT)还原 为产物胺的反应，虽然 Likhar 等没有直接检测到铜氢化 物的存在，他们认为该反应经过原位形成的 $[\mathrm{Cu}-\mathrm{H}]$ (monohydridocopper)的机理(应属典型借氢类型的机理) 进行, 形成的 $[\mathrm{Cu}-\mathrm{H}]$ 进一步将亚胺还原为胺. 该报道
中, Likhar 等没有将空气的影响考虑在内, 也没有进行 空气和惰性氛围下对照反应的实验.

同年, Shi 和 Beller 等 ${ }^{[18]}$ 报道了 $\mathrm{Cu}(\mathrm{OAc})_{2} / \mathrm{K}_{2} \mathrm{CO}_{3}$ 均 相体系催化的磺酰胺与芐醇在空气下的 $N$-烷基化反应 (Eq. 4). 该方法需使用过量的醇(4 equiv.)、较高的反应 温度 $\left(150{ }^{\circ} \mathrm{C}\right)$, 且底物范围有限, 仅适用于取代磺酰胺 和取代茮醇. 其中, 仲醇的反应需在无碱条件下进行, $\mathrm{Cu}(\mathrm{OTf})_{2}$ 的效果好于 $\mathrm{Cu}(\mathrm{OAc})_{2}$.

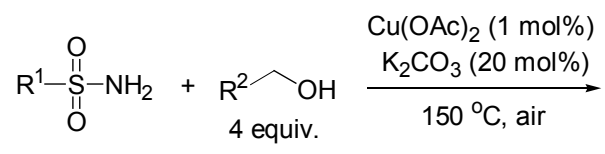

$$
\begin{array}{ll}
\mathrm{R}^{1}-\mathrm{M}_{\mathrm{H}} & \begin{array}{l}
\mathrm{R}^{1}=\text { alkyl, aryl; } \mathrm{R}^{2}=\text { aryl } \\
14 \text { examples } \\
89 \% \sim 97 \% \text { yields }
\end{array}
\end{array}
$$

与上述 Likhar 等 ${ }^{[17]}$ 的报道不同, Shi 等 ${ }^{[18,19]}$ 发现，在 空气下的反应其效果远好于惰性氛围下的反应(Eq. 5). 如 Eq. 5 所示, 在氩气和二氧化碳氛围下，反应的转化率 只有一半, 在空气下却接近完全. 该结果说明空气可以 大大促进反应的进行，但是 Shi 等没有对空气的作用进 行进一步的研究. 相反, 他们在空气下的反应中发现了 一种可能结构为 11 的杂质. 由于在反应中加入合成的 11 可在一定程度上促进反应的进行, Shi 等认为, 在空气 条件下，原位生成的 11 可能作为 $\mathrm{Cu}$ 催化剂的配体促进 反应的进行. 不过，由于在反应条件下 11 容易分解，11 只适用于一种底物组合(即对甲苯磺酰胺和苠醇), 否则 将导致混合产物的生成，结构相近而难于分离. 在反应 机理方面, Shi 等认为该反应中 $\mathrm{Cu}$ 催化剂对醇的活化效 果比较意外, 但是并没有去检测反应中的活性 $\mathrm{Cu}$ 催化 剂及其 $[\mathrm{Cu}-\mathrm{H}]$ 物种, 而是直接认为 $\mathrm{Cu}$ 催化的反应应该 与 $\mathrm{Ru}$ 催化的反应 ${ }^{[9,11]}$ 类似, 经过如 Scheme 9 所示的借 氢机理进行.

在之后的全文中, Shi 等 ${ }^{[20]}$ 对反应、实验现象和机理 研究进行了更详细的描述. 他们报道, 反应在空气或氧 气多了和少了的情况下都不好: 含有 $14 \mathrm{~mol} \%$ 的氧气时, 反应可达到最佳的效果; $70 \mathrm{~mol} \%$ 氧气时，目标产物产

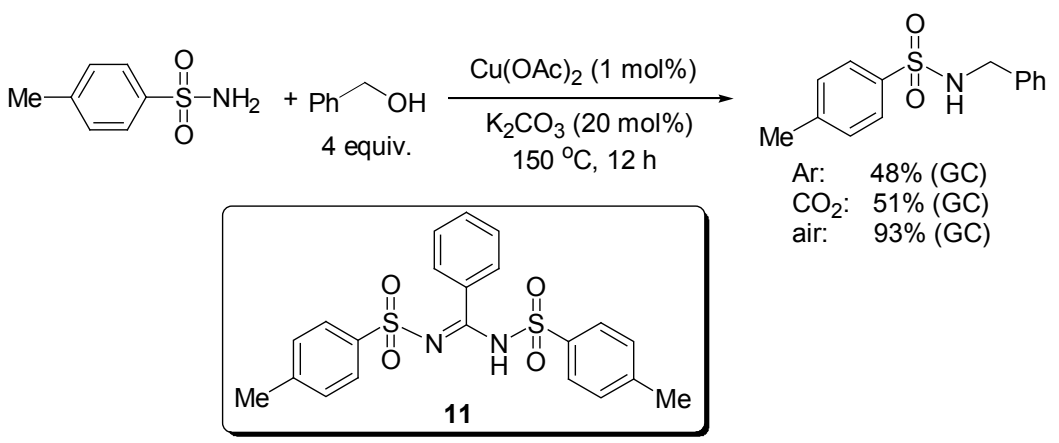




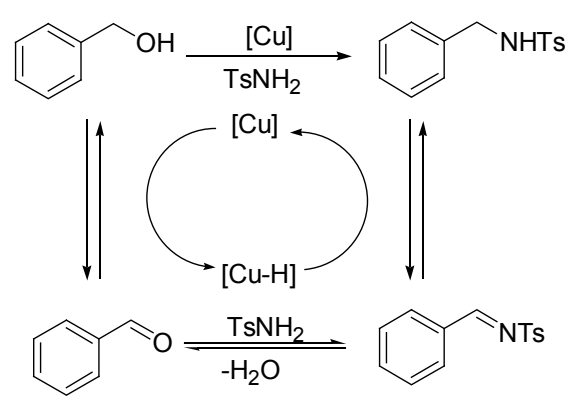

Scheme 9

率大大降低, 约为 $30 \%$, 但是生成了大量的亚胺中间体 (约 52\%). 通过研究氧气的量对反应的影响, 他们得出 结论, 空气导致了 11 的产生, 进而作为配体促进反应进 行. 在该全文中, Shi 等 ${ }^{[12]}$ 提出仲醇的反应更可能经过碳 正离子中间体的机理进行.

2009 年开始, Xu 课题组 ${ }^{[21]}$ 也着手研究醇与胺和酰 胺的脱水 $N$-烷基化反应以合成有用的胺和酰胺衍生物. 他们首先使用与 $\mathrm{Ir}, \mathrm{Ru}$ 类似的铑 $(\mathrm{Rh})$ 催化剂 $\mathrm{Rh}\left(\mathrm{PPh}_{3}\right)_{3} \mathrm{Cl}$ (即著名的 Wilkinson 催化剂) 催化磺酰胺与芐醇在无溶 剂条件下的 $N$-烷基化反应(Eq. 6). 他们意外的发现, 与 $\mathrm{Ir}$ 和 $\mathrm{Ru}$ 催化的反应不同, 该 $\mathrm{Rh}$ 催化的反应在氮气下 $120{ }^{\circ} \mathrm{C}$ 时根本不发生; 相反, 空气下的反应却可以非常 迅速地进行, 几乎当量地得到目标产物(96\%). 该 Rh 催 化的方法适用于芐醇、杂芐醇、烯丙醇以及磺酰胺、酰 胺和杂芳胺等一系列底物. 初步研究显示, Rh 催化剂、 碱和空气对反应快速地进行都起到至关重要的作用, 缺 一不可. 因此, $\mathrm{Xu}$ 等 ${ }^{[2]}$ 提出了一种区别于借氢机理的新 机理的可能性.

$$
\begin{gathered}
\mathrm{R}^{1} \widehat{\mathrm{OH}}+\mathrm{R}^{2}-\mathrm{NH}_{2} \frac{\mathrm{Rh}\left(\mathrm{PPh}_{3}\right)_{3} \mathrm{Cl}(2 \mathrm{~mol} \%), \mathrm{K}_{2} \mathrm{CO}_{3} \text { (1 equiv.) }}{\text { air, } 120^{\circ} \mathrm{C}, 12 \mathrm{~h}} \\
\mathrm{R}^{1}=\text { aryl, heteroaryl, allyl; } \mathrm{R}^{2}=\mathrm{RSO}_{2}, \mathrm{PhCO} \text {, heteroaryl } \\
19 \text { examples, } 67 \% \sim 99 \% \text { yiels }
\end{gathered}
$$

$\mathrm{Xu}$ 等 ${ }^{[22]}$ 后来对反应条件、催化剂和底物范围进行 了进一步的优化和拓展, 开发了一种普遍的空气促进的 过渡金属催化的有氧 $N$-烷基化方法(Eq. 7). 反应条件方 面，在空气下使用 $0.5 \mathrm{~mol} \%$ 的 Rh 催化剂、 $10 \mathrm{~mol} \%$ 的碱、

$$
\begin{gathered}
\mathrm{R}^{1>\mathrm{OH}}+\mathrm{R}^{2}-\mathrm{NH}_{2} \underset{\text { air, } 120 \sim 150^{\circ} \mathrm{C}, 4 \sim 48 \mathrm{~h}}{\text { base }(10 \sim 100 \mathrm{~mol} \%)} \\
\mathrm{M}=\underset{\mathrm{Rh}\left(\mathrm{PPh}_{3}\right)_{3} \mathrm{Cl}, \mathrm{RhCl}_{3} \cdot 3 \mathrm{H}_{2} \mathrm{O}, \mathrm{Rh}_{2} \mathrm{O}_{3}, \mathrm{Rh}_{2}(\mathrm{OAc})_{4},}{\mathrm{RuCl}_{3} \cdot \mathrm{nH}_{2} \mathrm{O}, \mathrm{RuO}_{2}, \mathrm{IrCl}_{3}, \mathrm{IrO}_{2} \text {, etc. }} \\
\mathrm{R}^{1}=\text { aryl, heteroaryl, alkyl; } \mathrm{R}^{2}=\mathrm{RSO}_{2}, \mathrm{RCO} \text {, heteroaryl, aryl } \\
91 \text { examples, } 58 \% \sim 99 \% \text { yields }
\end{gathered}
$$

1.3 equiv.的醇, 在 $135{ }^{\circ} \mathrm{C}$ 、无溶剂条件下, 即可获得高 收率的产物. 催化剂方面, 他们发现, 在空气下, 很多 无配体、简单、更经济易得的 $\mathrm{Rh}, \mathrm{Ru}$ 和 Ir 催化剂及其 氧化物都可以高效地催化反应. 底物方面, 该方法还可 以进一步扩展到脂肪醇和芳胺等. 与空气下的快速反应 形成鲜明对比, 很多氮气下进行的平行反应不发生或者 只得到低收率的产物. 虽然某些氮气下的反应可以通过 添加配体等方式提高收率(使用配体应属于典型的借氢 方法), 将反应在空气下进行显然是更好的选择, 起到了 跟添加配体同样的促进反应的效果.

$\mathrm{Xu}$ 等 ${ }^{[22]}$ 对该空气促进的过渡金属催化的有氧 $N$-烷 基化反应的机理也进行了较细致的研究. 他们首先排除 了反应经过碳正离子机理 ${ }^{[12]}$ 的可能性. 由于该反应在反 应条件、实验现象等多方面与典型无氧条件下的借氢反 应存在明显的区别, 与 Shi 等 ${ }^{[18,20]}$ 的观点不同, 他们认 为无氧和有氧两种类型的反应可能经过不同的分步反 应和机理进行(Scheme 10). 如 Scheme 10 所示, 与形成 $[\mathrm{MH}]$ 或 $\left[\mathrm{MH}_{2}\right]$ 的典型借氢反应不同(Path 1), 空气存在下 的有氧反应(Path 2)更可能经历空气下过渡金属催化的 有氧醇氧化、醛胺脱水缩合、亚胺被醇通过转移氢化反 应还原为胺而醇转化为醛三个过程进行.

在醇氧化一步 ${ }^{[23]}, \mathrm{Xu}$ 等首先证明, 在氮气下, 醇在 金属催化下转化为醛是一个慢速的反应，而该过程可以 被空气大大加速，即金属催化的空气对醇的氧化反应; 其次, 在磺酰胺存在下, 醇在氮气下不能被 $\mathrm{Rh}\left(\mathrm{PPh}_{3}\right)_{3} \mathrm{Cl}$ 脱氢, 在空气下却可以, 不仅再次说明空气所起的作用, 同时也说明胺或酰胺类化合物能与金属络合 ${ }^{[24,25]}$, 对金 属催化剂有一定的钝化甚至毒化作用 ${ }^{[24]} . \mathrm{Xu}$ 等 ${ }^{[26]}$ 后期 在钯 $(\mathrm{Pd})$ 催化的有氧 $N$-烷基化反应中报道了胺与钯的络 合现象 (Eq. 8), 进一步证实了胺与酰胺类化合物与金属 催化剂的络合作用.

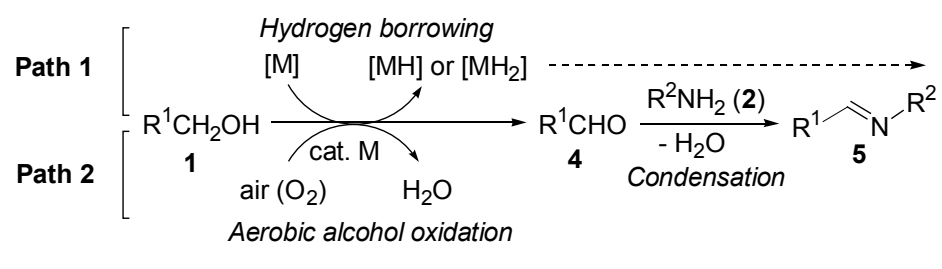

Scheme 10

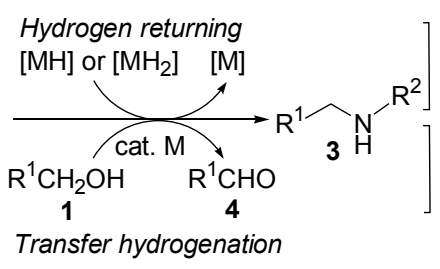




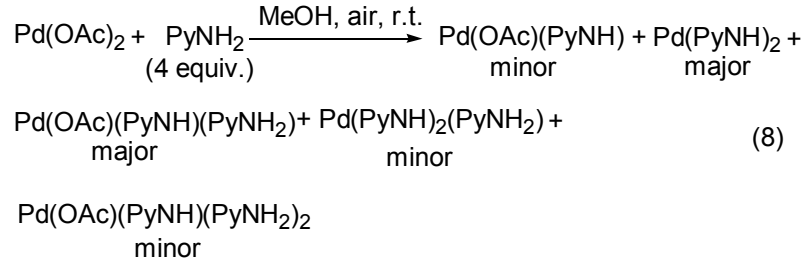

$\mathrm{Pd}(\mathrm{OAc})(\mathrm{PyNH})\left(\mathrm{PyNH}_{2}\right)_{2}$

minor

在醛与胺脱水所生成亚胺缩合反应一步, $\mathrm{Xu}$ 等通过 实验证明，过渡金属催化剂能够促进该反应的进行. 该 实验结果与 Crabtree 等认为过渡金属与醛的络合能增加 醛的亲核性、并促进缩合反应的预测 ${ }^{[11 \mathrm{~g}]}$ 是一致的; 相 反, 借氢理论认为, 醛与胺的缩合不需要过渡金属催化 剂的参与(Schemes 3 和 9$)^{[9,11]}$. 实际上, 很多路易斯酸和 金属催化剂被大量的用于亚胺的合成和反应中，可以大 大促进醛、酮与胺进行更有效的缩合 ${ }^{[2,27]}$.

在醇作为氢源还原亚胺为胺产物的转移氢化反应 一步, $\mathrm{Xu}$ 等通过核磁分析, 首次在醇作为氢源还原亚胺 的转移氢化反应中 ${ }^{[28]}$ 证实: 当量的羰基化合物可以在该 反应中得到再生(Eq. 9). 理论上, 再生的醛可以回收再 利用、与胺缩合, 完成催化循环.

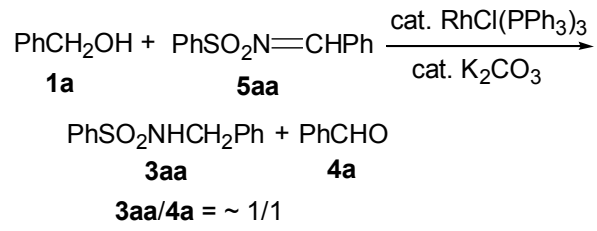

在上述一系列机理研究的基础上, $\mathrm{Xu}$ 等提出了该空 气促进的过渡金属催化的有氧脱水 $N$-烷基化反应研究 中不同于以往文献观点 ${ }^{[17 ~ 20]}$ 的机理 (Scheme 11). 同时, 这也是过渡金属催化脱水 $N$-烷基化反应研究中 ${ }^{[9,11]}$ 首次 提出的一种区别于借氢机理的新机理. 如 Scheme 11 所 示, $\mathrm{Xu}$ 等还对醇活化为醛(通过脱氢或氧化, 步骤 i)、醛 胺缩合(步骤 ii)、转移氢化(步骤 iii)三步反应进行了对比. 他们发现, 缩合和转移氢化反应均可在较低温度、无氧 的条件下快速地进行, 而醇的活化由于受催化剂针化等 因素的影响成为催化循环中的决速步骤. 因此, 只有醇 活化一步(步骤 $\mathrm{i}$ )需要空气的参与来降低反应的温度.

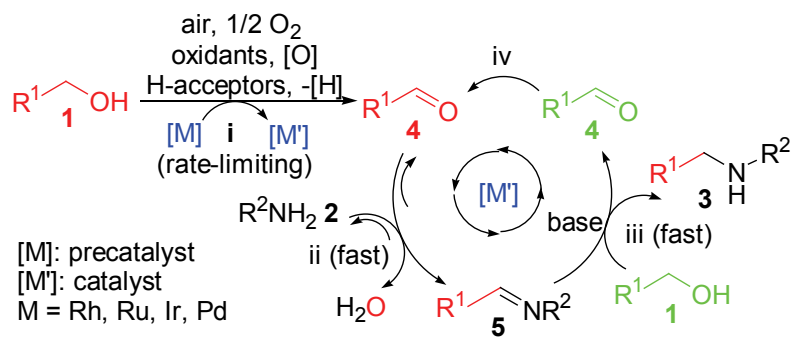

Scheme 11

由此机理可以看出, 通过醇氧化(步骤 i)生成的醛消
耗之后，可以通过转移氢化反应(步骤 iii)中醛的生成得 到补充, 即转移氢化一步生成的副产物一醛, 可以在反 应中得到很好的回收利用(步骤 iv). 因此，整个反应只 需催化量的空气即可快速进行，这与实验现象相符. 由 此机理也可以推论, 在惰性氛围下, 通过加入某些氧化 剂或者脱氢试剂以促进醇脱氢、或者通过添加醛、亚胺 等中间体，也可以促使反应有效地进行，Xu 等也通过实 验证实了这些预测. 显然，直接将反应在空气下进行是 既好又简便的选择。

$\mathrm{Xu}$ 等提出的新机理(Scheme 11), 不仅可以解释空 气下的反应中一些特殊的现象、回答之前没有得到合理 解释的一些疑问，还可以有效地对反应进行一些预测. 对比 Likhar 和 Shi 等 ${ }^{[17,18,20]}$ 的报道, Xu 等的研究更为细 致和透彻, 相关分步反应和机理研究不仅经过实验证 实，也有其他相关研究领域大量的文献支持(见上下 文) ${ }^{[2,4,13 \sim 16,23 \sim 25,27,28]}$. 因此, 他们提出的机理也是有关有 氧 $N$-烷基化反应中更合理的机理.

由于前一分子的醇在转变为醛并与胺缩合为亚胺 后，将从后一分子的醇中获得氢，而后一分子的醇也将 在下一循环中再从后一分子的醇中获得氢. 这一过程非 常类似于接力赛中接力棒的传递, 因此 Xu 等将此机理 形象的描述为“接力”机理.

$\mathrm{Xu}$ 等后来也将上述有氧 $N$-烷基化方法扩展到了无 配体的均相 $\mathrm{Pd}$ 催化剂, 报道了低用量 $\mathrm{Pd}$ 催化的有氧脱 水 $N$-烷基化反应(Eq. 10) ${ }^{[26]}$. 与文献报道的非均相 $\mathrm{Pd}$ 催 化的方法 ${ }^{[29]}$ 相比，该均相 Pd 催化的方法相对简便、高 效、产物选择性高. 机理研究显示, 该反应与 $\mathrm{Rh}, \mathrm{Ru}, \mathrm{Ir}$ 催化的反应类似，因此也应通过接力机理进行 (Scheme 11). 该研究结果不仅进一步支持接力机理，也拓展了接 力方法的催化剂范围.

$$
\begin{aligned}
& \mathrm{R}^{1} \widehat{\mathrm{OH}}+\mathrm{R}^{2}-\mathrm{NH}_{2} \underset{\text { cat. base, under air, } 135^{\circ} \mathrm{C}, 24 \sim 48 \mathrm{~h}}{\longrightarrow} \\
& {[\mathrm{Pd}]=\mathrm{Pd}(\mathrm{OAc})_{2}, \mathrm{PdCl}_{2} \text {, etc }} \\
& \mathrm{R}^{1} \widehat{\mathrm{N}} \mathrm{H}^{-\mathrm{R}^{2}}+\mathrm{H}_{2} \mathrm{O}
\end{aligned}
$$

在 $\mathrm{Xu}$ 等 ${ }^{[21,22]}$ 的报道之后, Ramón 等 ${ }^{[30]}$ 也报道了空 气下均相 $\mathrm{Pd}$ 催化的磺酰胺的 $N$-烷基化反应(Eq. 11). 他 们的方法使用更低用量的 $\mathrm{Pd}$ 催化剂(0.05 mol\%), 但是 需使用当量的无机碱、溶剂和较高的反应温度. 与上述 几例报道类似，Ramón 等发现，在磺酰胺的反应中，空 气下的反应效果好于氩气下的反应. 虽然如此，他们没 有对反应的机理进行研究，而是直接认为该反应经过他 们提出的氢自动转移机理 ${ }^{[9,11 \mathrm{~h}]}$ 进行. 


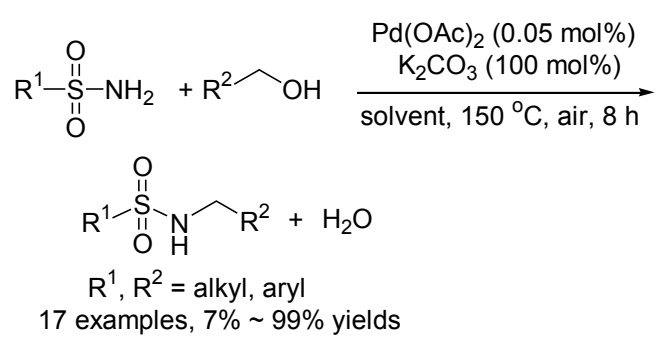

同年, Fujita 和 Yamaguchi 等 ${ }^{[31]}$ 报道了水溶性 Ir 催 化剂 12 催化的可在空气下进行的醇与胺的 $N$-烷基化反 应(Eq. 12). 该报道对一般有机溶剂中进行的借氢反应 进行改进, 使用更为绿色的水为溶剂. 由于催化剂 12 较 稳定，反应可在空气下进行. 该方法适用于一系列芳 胺、脂肪胺、伯胺、仲胺以及芐醇、脂肪醇、伯醇、仲 醇等等, 因此可从伯胺制备仲胺和叔胺, 也可从仲胺制 备叔胺. Fujita和 Yamaguchi 等对催化剂的回收利用也进 行了考察, 12 经过三次循环使用, 催化活性没有明显降 低.

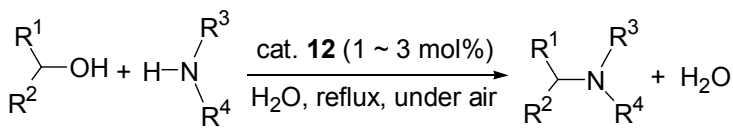

$$
\begin{aligned}
& \mathrm{R}^{1}, \mathrm{R}^{2}=\text { alkyl, aryl, } \mathrm{H} ; \mathrm{R}^{3}, \mathrm{R}^{4}=\text { alkyl, aryl, } \mathrm{H} \\
& 37 \text { examples, } 74 \% \sim 94 \% \text { yields } \\
& \underbrace{2+2 \mathrm{I}^{-}}_{12}
\end{aligned}
$$

Fujita 和 Yamaguchi 等对反应机理中的转移氢化一 步进行了研究, 但是没有考察空气对反应的影响. 根据 机理实验结果和自身的研究经验, 他们认为反应经过类 似的活性 $[\mathrm{Ir}-\mathrm{H}]$ 物种参与的氢转移机理 ${ }^{[11 i]}$ 进行. 简化的 机理如 Scheme 12 所示. 在该机理中, Fujita 等也考虑到 了胺与 $\mathrm{Ir}$ 催化剂的络合作用 ${ }^{[25]}$.

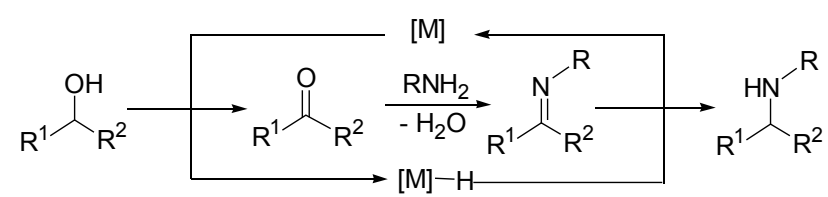

\section{Scheme 12}

稍后于 Fujita 和 Yamaguchi 等的报道, Uozumi 和 Yamada 等 ${ }^{[32]}$ 在 2011 年也报了空气下水相中多聚 [PB-Cp ${ }^{*} \mathrm{Ir}(\mathrm{IPr})$ ]双金属催化剂 13 催化的氨水和胺与醇的 脱水 $N$-烷基化反应(Scheme 13). 该方法适用于芐醇、脂 肪醇和芳胺的反应. 不同的是, 芐胺、脂肪胺、仲胺以 及氨水与苠醇的反应需在 $\mathrm{pH}=4$ 的缓冲液及微波辐射 辅助的条件下进行. 虽然Uozumi 和 Yamada 等没有该反
应的机理进行研究, 他们认为, 采用水溶性非均相催化 剂在水相中实现脱水 $N$-烷基化反应是对常规借氢反应 的改进.

$$
\begin{aligned}
& \text { aq. } \mathrm{NH}_{3}+\mathrm{HOR} \frac{\mathrm{PB}-\mathrm{Cp} \mathrm{p}^{*} \mathrm{Ir}(\mathrm{IPr})(1 \mathrm{~mol} \% \mathrm{Ir})}{\mathrm{H}_{2} \mathrm{O} \text {, under air }} \mathrm{NR}_{3} \\
& \mathrm{R}^{1} \mathrm{NH}_{2}+\mathrm{HOR} \underset{\mathrm{PB}-\mathrm{Cp}{ }^{*} \mathrm{Ir}(\mathrm{IPr})(1 \mathrm{~mol} \% \mathrm{Ir})}{\mathrm{H}_{2} \mathrm{O} \text {, under air }} \mathrm{R}^{1} \mathrm{NHR}\left(\mathrm{R}^{1} \mathrm{NR}_{2}\right) \\
& \mathrm{R}^{1} \mathrm{R}^{2} \mathrm{NH}+\mathrm{HOR} \underset{\mathrm{H}_{2} \mathrm{O} \text {, under air }}{\stackrel{\text { PB }}{*} \operatorname{li}(\mathrm{IPr})(1 \mathrm{~mol} \% \mathrm{Ir})} \mathrm{R}^{1} \mathrm{R}^{2} \mathrm{NR} \\
& \mathrm{R}=\text { benzyl, alkyl } \\
& \mathrm{R}^{1}, \mathrm{R}^{2}=\text { aryl, } \mathrm{H} \text {; alkyl, } \mathrm{H} \text {; alkyl, alkyl } \\
& 22 \text { examples, } 61 \% \sim 95 \% \text { yields } \\
& \overbrace{13}
\end{aligned}
$$

在上述贵重金属催化的有氧 $N$-烷基化反应 ${ }^{[21,22,26]}$ 的 基础上, 2011 年, $\mathrm{Xu}$ 等 ${ }^{[33]}$ 报道了空气促进的 $\mathrm{MnO}_{2}$ 催化 的醇与胺和磺酰胺在无溶剂条件下的脱水 $N$-烷基化反 应(Eq. 13). 机理研究显示, 空气对反应有促进作用, 因 而该反应也应经历接力机理进行(Scheme 11). 实验证 实, 在醇活化一步(Eq. 14), 氮气下可能经过 $\mathrm{Mn}(\mathrm{IV})$ 和 $\mathrm{Mn}(\mathrm{II})$ 转变的慢速活化过程; 在空气下, 通过 $\mathrm{Mn}$ 的催 化作用, 空气起到加速醇活化的关键作用. 与文献上报 道的简单非均相金属氧化物 ${ }^{[3]}$ 或负载在简单金属氧化 物上的贵金属催化剂 ${ }^{[29,35]}$ 催化的脱水 $N$-烷基化反应相 比, 直接利用简单、廉价的 $\mathrm{MnO}_{2}$ 为催化剂的反应, 无需 苛刻的反应条件(比如高温、强碱、大大过量的醇或胺), 反应在空气下就可以得到很好的反应效果, 产物的产率 和选择性普遍较好, 而且还可以避免使用贵重金属和非 均相金属催化剂的制备，因此是一种更经济绿色的方 法.

$$
\begin{aligned}
& \mathrm{R}_{\mathbf{1}}^{1} \widehat{\mathrm{OH}}_{\stackrel{2}{\mathrm{R}^{2}} \mathrm{NH}_{2}}^{\stackrel{\text { ligand-free } \mathrm{MnO}_{2}(10 \sim 20 \mathrm{~mol} \%)}{\text { cat. base, under air, neat }}} \\
& \text { air } \gg>\mathrm{N}_{2}, 35 \text { examples, up to } 99 \% \text { yield }
\end{aligned}
$$

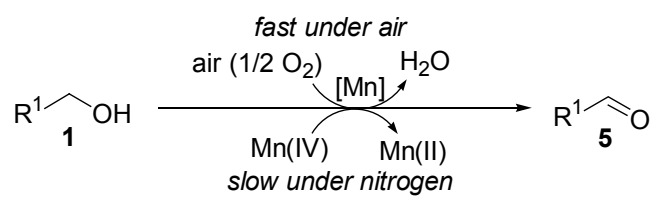

$\mathrm{Xu}$ 等的上述研究结果也显示, 除了借氢反应中的 无氧脱氢方式外，同时也类似于 $\mathrm{Li}$ 等提出的氧化剂(包 括氧气和空气)作用下胺类和醚类化合物脱氢活化的交 
叉脱氢偶联反应(cross-dehydrogenative coupling $)^{[36]}$, 空 气下金属催化的有氧醇氧化也可以是一种新的醇的活 化方式(Schemes 10 和 11). 这种醇活化方式, 还可以因 为空气的参与和水的生成拉动活化反应、降低反应能垒 和反应条件, 促进反应在较低温度下进行. 因此, 理论 上，良好的醇氧化催化剂极有可能也是良好的有氧 $N$-烷 基化反应的催化剂. 因而, 可以采用优良的廉价金属催 化剂代替贵重金属催化剂实现反应成本的降低和反应 规模的扩大. 由文献可知, $\mathrm{Cu}$ 是优良的醇氧化催化 剂 ${ }^{[23,37]}$ 和 $\mathrm{C}-\mathrm{H}$ 活化-氧化偶联反应中的氧化剂和催化 剂 ${ }^{[38]}$. 虽然 Likhar, Shi 和 Yus 等 ${ }^{[17 \sim 20]}$ 已经报道了一些均 相或非均相、有氧或无氧的 $\mathrm{Cu}$ 催化的 $N$-烷基化反应, $\mathrm{Xu}$ 等 ${ }^{[39]}$ 在 2012 年也报道了持不同观点的 $\mathrm{Cu}$ 催化的有氧脱 水 $N$-烷基化反应研究.

$\mathrm{Xu}$ 等采用更便宜易得、稳定的水合醋酸铜 $\mathrm{Cu}(\mathrm{OAc})_{2} \cdot \mathrm{H}_{2} \mathrm{O}$ 为催化剂, 优化得到比已报道的 $\mathrm{Cu}(\mathrm{OAc})_{2}$ 催化的其他方法 ${ }^{[18 ~ 20]}$ 更佳的反应条件. 新方 法使用更少量的醇(1.3 equiv.)和碱(10 mol\%), 在空气下 $135{ }^{\circ} \mathrm{C}$ 就实现了高效的有氧脱水 $N$-烷基化反应(Eq. 15). 该反应不限于磺酰胺, 还适用于一系列的杂芳胺、芳胺, 拓展了 $\mathrm{Cu}$ 催化方法的底物范围.

$$
\begin{aligned}
& \mathrm{R}_{\mathrm{OH}}^{1}+\mathrm{R}^{2} \mathrm{NH}_{2} \stackrel{\begin{array}{c}
\mathrm{Cu}(\mathrm{OAc})_{2} \cdot \mathrm{H}_{2} \mathrm{O}(1 \mathrm{~mol} \%) \\
\text { base }(10 \sim 20 \mathrm{~mol} \%)
\end{array}}{\text { air, } 135{ }^{\circ} \mathrm{C}, 24 \sim 36 \mathrm{~h}} \\
& \mathrm{R}^{1} \frown \mathrm{N}^{-} \mathrm{R}^{2}+\mathrm{H}_{2} \mathrm{O} \\
& \mathrm{R}^{2}=\mathrm{RSO}_{2}, \text { Aryl, heteroaryl } \\
& 29 \text { examples, } 19 \% \sim 95 \% \text { yields }
\end{aligned}
$$

反应机理方面, $\mathrm{Xu}$ 等通过一系列机理研究以及文献 依据 ${ }^{\left[{ }^{[37}, 38,40\right]}$ 认为, 任何条件下, 该反应生成和经过铜氢 化物中间体 $[\mathrm{Cu}-\mathrm{H}]$ 的机理的可能性都不大. 首先, $[\mathrm{Cu}$ - $\mathrm{H}$ ]的生成需要在情性氛围下强给电子的配体(如膦配 体)和强的氢供体(如硅氢试剂)的存在, 而且非常敏感活 泼、易于被破坏 ${ }^{[40]}$. 因此, 有氧条件下几乎不可能生成 $[\mathrm{Cu}-\mathrm{H}]$ 物种. 假设反应中可形成 $[\mathrm{Cu}-\mathrm{H}]$ 物种, 应具有 强还原性, 那么惰性氛围下的反应理应好于空气下的反 应, 这与实验现象不符, 因此也可以排除 $[\mathrm{Cu}-\mathrm{H}]$ 生成 的可能性. 其次, 实验现象表明, 在氮气下, $\mathrm{Cu}(\mathrm{II})$ 与醇 作用生成酫不仅需要较高的反应温度 $\left(>150{ }^{\circ} \mathrm{C}\right)$, 而且, 与加入的 $\mathrm{Cu}(\mathrm{II})$ 成定量关系的苯甲醛 $(\mathbf{4 a})$ 及醋酸茮酯可 以被核磁较准确地检测到(Eqs. 16 18). 由于发现 $\mathrm{Cu}(\mathrm{I})$ 不起氧化醇的作用, 因此可以认为: 在情性氛围下, 醇 被 $\mathrm{Cu}(\mathrm{II})$ 氧化成醛, $\mathrm{Cu}(\mathrm{II})$ 转化为 $\mathrm{Cu}(\mathrm{I})$. 该结果也可以很 好地解释, 在惰性氛围下, $\mathrm{Cu}(\mathrm{II})$ 对反应较弱的催化作

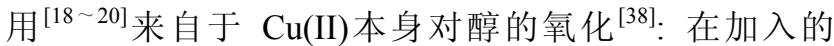
$\mathrm{Cu}$ (II)为 $1 \mathrm{~mol} \%$ 的情况下, 只产生极少量的醛, 因此, 惰性氛围下的反应需较高的温度和较长的时间 ${ }^{[18-20]}$.

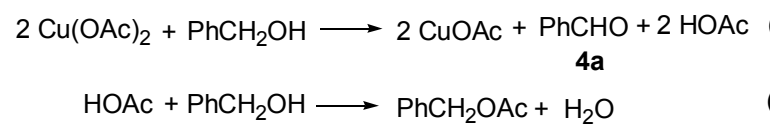

Overall reaction (Eqs. $16+17)$ :

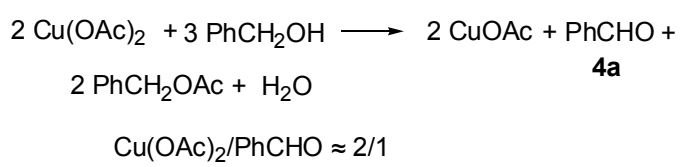

相反, 由于醇活化为醛是整个反应的决速步, 在空 气下, 空气参与醇氧化对醇活化的加速可以促进整个反 应更快速的进行，这点也在使用被不同浓度的醛污染的 醇的对照实验中得到了很好的证实. 因此 Xu 等也分析, Shi 等认为的起配体作用而促进反应进行的杂质 $\mathbf{1 1}^{[18,20]}$, 很有可能是因为 11 的不稳定, 继而在反应条件下分解 出醛或者亚胺的方式促进反应进行的. 此外，与 Shi 等 观察到的现象 ${ }^{[20]}$ 相同，在大量空气的存在下, $\mathrm{Xu}$ 等也在 反应中检测到了大量的亚胺. $\mathrm{Xu}$ 等认为, $\mathrm{Cu}$ 催化醇氧化 成醛 ${ }^{[23,37]}$ 再与胺的缩合反应 ${ }^{[27]}$ 是导致亚胺生成的原因, 这点也在后来的醇与胺的有氧氧化亚胺合成方法研究 中进一步得到了确证(见下文) ${ }^{[41,42]}$. 总之, $\mathrm{Xu}$ 等在该报 道中的这些实验证据很好地解释了 $\mathrm{Cu}(\mathrm{II})$ 和空气促进反 应的真正原因，也对机理中的各个步骤进行了实验证 明，因此该反应也应该通过接力机理进行(Scheme 14).

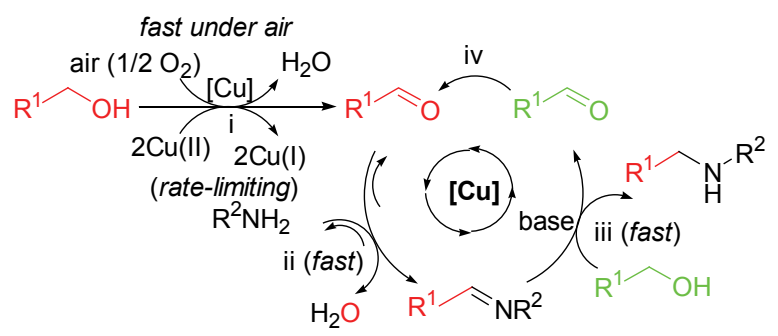

\section{Scheme 14}

\section{3 醇与仲醇和甲基酮的有氧 $C$-烷基化反应}

与醇在胺和酰胺类化合物的脱水 $N$-烷基化反应中 的应用类似，一些含活泼 $\mathrm{C}-\mathrm{H}$ 键的化合物(典型的比如 仲醇、甲基酮)也能发生类似的脱水 $C$-烷基化反应. 目 前, 大部分脱水 $C$-烷基化反应研究主要使用均相或非均 相 $\mathrm{Ru}, \mathrm{Ir}, \mathrm{Pd}, \mathrm{Au}, \mathrm{Ag}$ 等贵重金属催化剂在惰性氛围下进 行 $^{[11]}$. 一般认为, 这些反应也通过借氢或氢自动转移机 理(Scheme 3)进行. 然而, 在已知的反应中, 为活化金属 催化剂, 往往需加入大量的脱氢试剂和碱, 有些反应的 选择性不是很高. 因此, 开发改进的方法或者使用廉价 
且高效的催化剂用于脱水 $C$-烷基化反应值得进一步研 究.

目前, 也有一些空气下进行的脱水 $C$-烷基化反应的 研究报道. 2006 年, Uozumi 等 ${ }^{[43]}$ 报道了首例在空气下进 行的甲基酮(7)与伯醇的有氧脱水 $\alpha$ - $C$-烷基化反应(Eq. 19). 该反应使用制备的 nano-Pd-V 为催化剂、 1 equiv. 氢氧化钡为碱, 2 equiv. 的伯醇与甲基酮在空气下加热 24 $h$, 可得良好到高收率的产物 9. 该方法适用于脂肪族和 芳香族甲基酮以及脂肪族伯醇和苠醇. Uozumi 等没有对 反应机理进行研究, 虽然他们认为反应理应经过氢转移 机理 ${ }^{[11]}$ 进行, 但是鉴于氢金属物种 $[\mathrm{MH}]$ 易于与氧反应, Uozumi 等认为其他类型的机理也可能参与了反应.

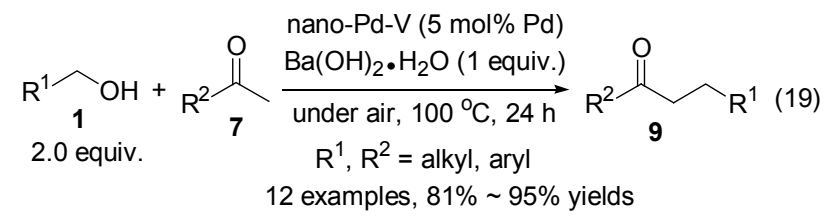

在后续的全文中 ${ }^{[44]}$, Uozumi 等对催化剂的回收利 用进行了考察, 发现其催化活性没有明显降低. 反应机 理方面, Uozumi 等并未像预期的那样提出其他可能的机 理, 而是仍然提出了尚未得到完全证实的属于借氢类型 的反应机理(Scheme 15).

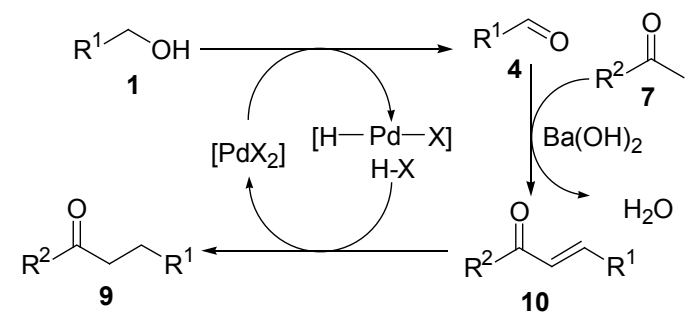

Scheme 15

2010 年, Crabtree 等 ${ }^{[45]}$ 报道了无过渡金属催化剂参 与的、空气下、碱作用的仲醇(6)的 $\beta-C$-烷基化反应(Eq. $20)$. 使用 1 equiv. 的碱、甲苯为溶剂, 将反应混合物在敞 口条件下加热回流的简单操作即可实现烷基化产物 8 和 9 的合成. 但是该方法底物范围较窄, 只能用于苠醇和 芳基仲醇，脂肪伯醇的反应收率很低(10\%～28\%), 而且 收率不错的反应往往目标产物 8 的选择性不高, 有的甚 至以副产物一烷基化的酮 9 为主.

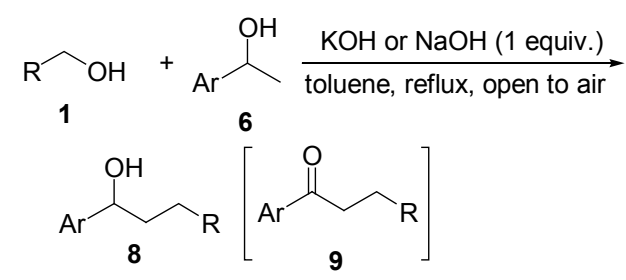

13 examples, $10 \% \sim 99 \%$ yields, $8 / 9=42 / 58 \sim 95 / 5$
Crabtree 等认为由于未入加金属催化剂, 该反应可 能不经过借氢机理进行, 而是通过其他的路径进行, 如 Scheme 16 所示, Crabtree 等推测: 首先是空气对醇的氧 化生成醛 4 和酮 7, 其次是碱作用下的差醛缩合生成 $\alpha, \beta$ 不饱和酤 10 , 接着 10 经 MPV 过程还原得到取代醇 8 或 酮 9 产物. 由于以上假设缺乏实验数据的有力支持, Crabtree 等提出的反应路径, 还有待进一步通过实验证 实. 此外, 底物和试剂中含有的少量过渡金属不纯物的 检测和鉴定、及其对反应催化效果影响的研究，是当前 在过渡金属催化和无过渡金属参与的反应研究中受到 广泛关注的一个焦点 ${ }^{[46]}$. 因此, 这一报道中底物和碱中 残留过渡金属的检测及其对反应的影响，也有待通过进 一步的研究确定.

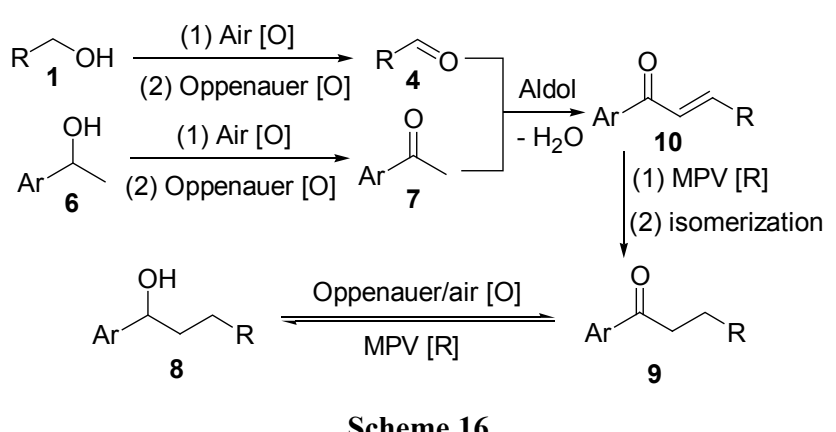

2011 年, Tang 和 Cheng ${ }^{[47]}$ 报道了 $\mathrm{Ni}$ 催化的乳酸 $\mathbf{1 4}$ 与伯醇合成 $\alpha$-差基羧酸 15 的脱水 $C$-烷基化反应(Eq. 21). 研究发现, 反应在无溶剂条件下较好, 且无需配体, 反应在氮气下和空气下的效果相近，因此反应可以方便 地在空气下进行. 温度对反应影响较大, 在 $140{ }^{\circ} \mathrm{C}$ 以下, 反应收率很低. 为使反应顺利进行, 该方法需使用较大 量的碱和较高的反应温度. 该报道也提供了一种 $\mathrm{Ni}$-催 化的仲醇的 $\beta$-C-烷基化方法(Eq. 22). 该反应需氮气保
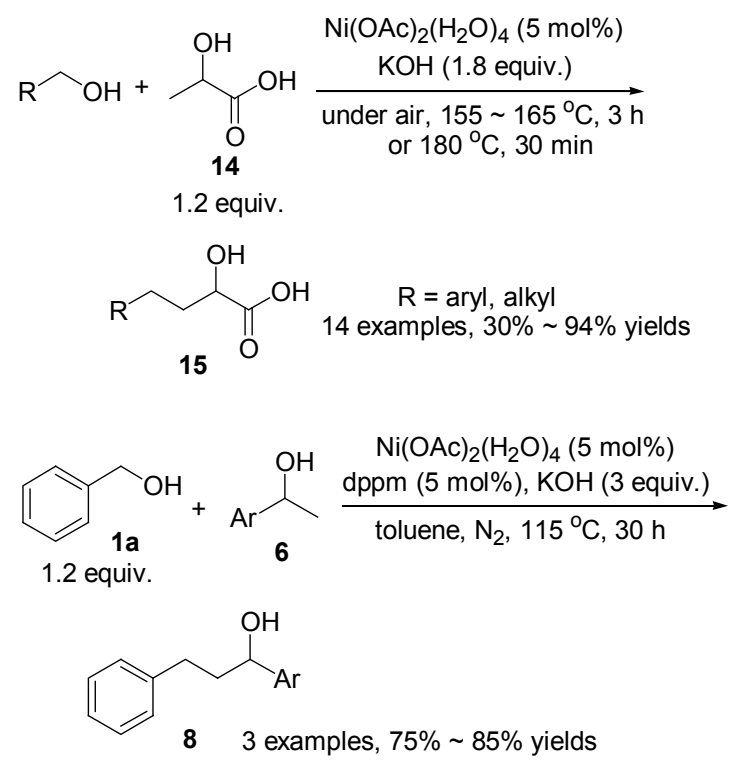
护、使用 $\mathrm{dppm}$ 为配体、过量的碱、溶剂和较长的反应 时间，目前的底物只限茮醇 $1 \mathrm{a}$ 与芳基仲醇. 该研究没有 报道证明反应机理的有关实验，但是认为该空气下乳酸 与伯醇的脱水 $C$-烷基化反应可能经过形成 $[\mathrm{H}-\mathrm{NiX}]$ 物种 的机理进行.

2012 年, 在报道 $\mathrm{Cu}$ 催化有氧 $N$-烷基化反应 ${ }^{[39]}$ 的同 时, $\mathrm{Xu}$ 等 ${ }^{[48]}$ 也报道了空气下 $\mathrm{Cu}$ 催化的仲醇 6 和甲基酮 7 与醇的有氧脱水 $C$-烷基化反应(Eqs. 23，24). 研究发 现，使用无配体的 $\mathrm{Rh}, \mathrm{Ru}$ 和 $\mathrm{Ir}$ 催化剂在氮气和空气下反 应效果都不好, $\mathrm{Pd}(\mathrm{OAc})_{2}$ 和 $\mathrm{Cu}(\mathrm{OAc})_{2}$ 的催化效果相对较 好, 其中以 $\mathrm{Cu}(\mathrm{OAc})_{2}$ 在空气下的反应为最好. 反应条件 经优化后，只使用 $1 \mathrm{~mol} \%$ 的 $\mathrm{Cu}(\mathrm{OAc})_{2} \cdot \mathrm{H}_{2} \mathrm{O}$ 、催化量的 $\mathrm{KOH}(30 \mathrm{~mol} \%)$, 在 $120{ }^{\circ} \mathrm{C}$ 反应 $24 \mathrm{~h}$ 即可以拿到高收率 和高选择性的目标产物 8 (Eq. 23). 该反应条件适用于 一系列的苠醇、杂苠醇、脂肪伯醇、芳基仲醇、脂肪族 仲醇. 脂肪醇参与的反应需要较高的温度和较长的时间 $\left(160{ }^{\circ} \mathrm{C}, 48 \mathrm{~h}\right)$. 该方法还适用于二级醇 6 自身的脱水 $C$ 烷基化反应，可得到良好收率相应产物. 类似的方法还 适用于甲基酮 7 的 $\alpha$ - $C$-烷基化反应(Eq. 24). 在使用过量

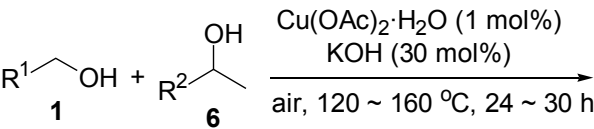

$$
\begin{aligned}
& \overbrace{8}^{\mathrm{OH}} \mathrm{R}^{1}[\mathrm{R}_{\mathrm{R}^{2}}^{\mathrm{O}} \overbrace{\mathrm{R}^{1}}] \\
& \mathrm{R}^{1}, \mathrm{R}^{2}=\text { aryl, heteroaryl, alkyl }
\end{aligned}
$$$$
25 \text { examples, } 26 \% \sim 99 \% \text { yields, sel. up to }>99 / 1
$$
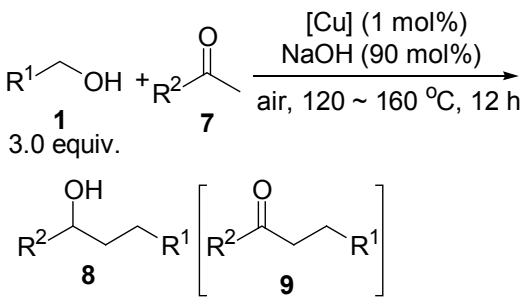

$$
\mathrm{R}^{1}, \mathrm{R}^{2}=\text { aryl, alkyl }
$$

9 examples, $74 \% \sim 99 \%$ yields, sel. up to $96 / 4$
伯醇的情况下，可以拿到良好到高收率的支链仲醇产物 8. 在此反应中, $\mathrm{NaOH}$ 的效果好于 $\mathrm{KOH}$.

与上述其他有氧 $C$-烷基化反应中模凌两可、未经实 验完全证实的机理表述 ${ }^{[43 ~ 45,47]}$ 不同, $\mathrm{Xu}$ 等对 $\mathrm{Cu}$ 催化的 有氧 $C$-烷基化反应机理进行了较细致的研究. 首先，与

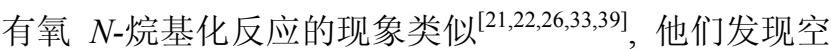
气同样对该 $C$-烷基化反应起到促进作用. 其次, 在醛 4 与酤 7 的羟醛缩合生成 $\alpha, \beta$-不饱和酮 $\mathbf{1 0}$ 的一步，他们发 现 $\mathrm{Cu}$ 催化剂同样可以大大促进反应进行(Eq. 25). 该结 果再次说明借氢类型机理中认为的金属对脱水缩合一 步不起作用的观点过于片面，也说明借氢机理本身还需 进一步完善. 此外, 他们还通过核磁研究发现, 在 $\alpha, \beta$ 不饱和酮 10 与醇的转移氢化反应中, 醇提供的氢可被 10 当量的吸收, 10 转化为主副产物 8 和 9 , 而醇 16 转化 为当量的相应的羰基化合物 17 (Eq. 26). 该结果也是在 $\alpha, \beta$-不饱和酮与醇的转移氢化反应中 ${ }^{[9,11]}$, 首次观察到 当量羰基化合物的产生. 根据一系列机理实验的结果, $\mathrm{Xu}$ 等为该 $\mathrm{Cu}$ 催化有氧 $C$-烷基化反应提出了类似于相 应有氧 $N$-烷基化反应的接力机理(Scheme 17).

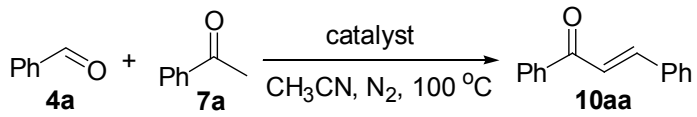

\begin{tabular}{cccc}
\hline Run & cat. $[\mathrm{Cu}](\mathrm{mol} \%)$ & 10aa (GC yield based on 7a) \\
\hline 1 & None & $6 \mathrm{~h}: 0.9 \% ;$ & $12 \mathrm{h:} 1.4 \%$ \\
2 & $\mathrm{Cu}(\mathrm{OAc})_{2} \bullet \mathrm{H}_{2} \mathrm{O}(5)$ & $6 \mathrm{~h}: 75 \% ;$ & $12 \mathrm{h:} 87 \%$ \\
\hline
\end{tabular}
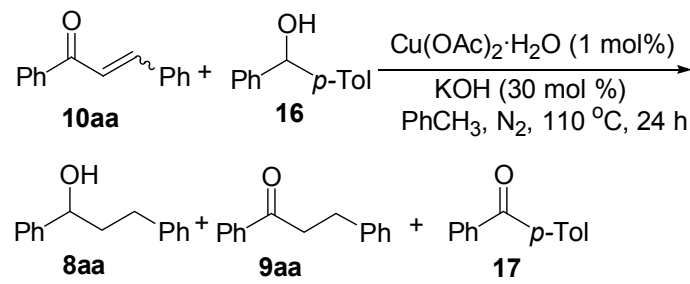

\begin{tabular}{ccccc}
\hline Run & 16/equiv. & 8aa/9aa $^{\text {NMR }}$ & 17\% $^{\text {NMR }}$ & $\left.\left(\mathbf{4}^{*} \mathbf{8 a a}+\mathbf{2}^{*} \mathbf{9 a a}\right) / \mathbf{2}^{*} \mathbf{1 7}\right)^{\mathrm{NMR}}$ \\
\hline 1 & 1.0 & $26 / 74$ & 84 & $0.97 / 1.00$ \\
2 & 3.0 & $61 / 39$ & 54 & $0.75 / 1.00$ \\
\hline
\end{tabular}

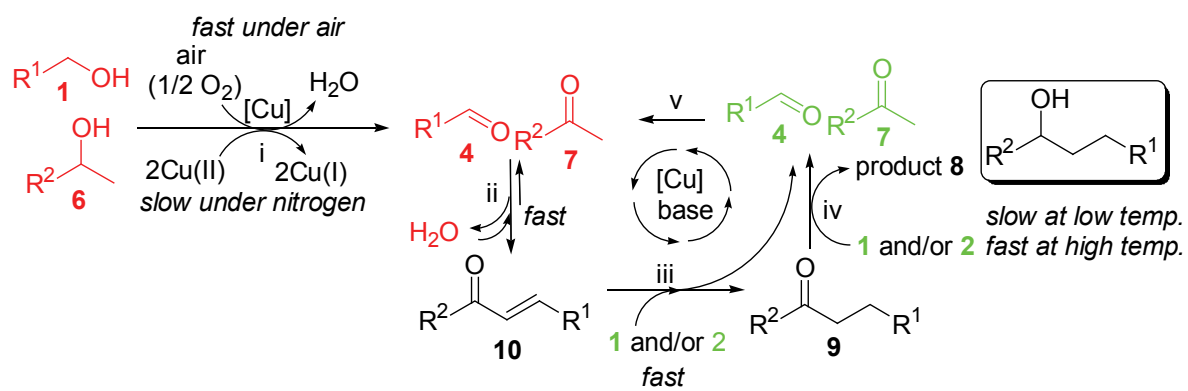

Scheme 17 


\section{2 醇与胺的有氧氧化缩合反应}

如前所述, 含有活泼 $\mathrm{C}=\mathrm{N}$ 双键的亚胺类化合物可 发生各种类型的转变, 是有机和药物合成等各个领域非 常重要的一类氮源和合成中间体 ${ }^{[2]}$. 因此, 各类亚胺化 合物的高效绿色合成研究仍然是当前亚胺化学的中心 内容之一[49,50].

亚胺也称席夫碱(Shiff base) ${ }^{[51]}$, 通常由胺与醛酮的 脱水缩合反应制备 ${ }^{[27]}$. 然而, 醛类化合物具有一定毒性 和臭味、易变质、易被氧化成酸、不易存储、使用前需 纯化. 由于醛酮一般可通过醇的氧化制备, 而且醇是最 容易获取、来源最广、价格最低、毒性较小又非常稳定 的有机化合物, 被视为较绿色合成试剂之一 ${ }^{[9,11,12 \mathrm{a} \sim \mathrm{b}]}$. 冊庸置疑, 直接使用醇为亚胺合成的原料(Scheme 18), 与传统方法相比具有很多优点, 也是在最近几年才发展 起来研究新方向. 该方法将醇氧化和醛与胺的缩合两步 反应在一锅条件下进行, 可以减少反应步骤、避免中间 产物的分离、降低消耗、提高反应效率和总产率. 目前, 该反应主要有使用当量氧化剂、脱氢缩合和有氧氧化缩 合三类方法.

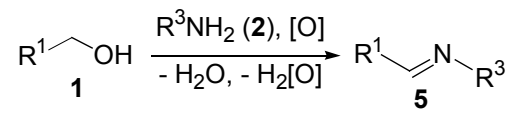

$$
\begin{aligned}
& \mathrm{R}_{1}^{1} \stackrel{\mathrm{R}}{2}_{\mathrm{OH}} \frac{\mathrm{R}^{3} \mathrm{NH}_{2}(\mathbf{2}),[\mathrm{O}]}{-\mathrm{H}_{2} \mathrm{O},-\mathrm{H}_{2}[\mathrm{O}]} \underset{\mathrm{R}^{2}}{\mathrm{R}_{5}^{1}}=\mathrm{N} \mathrm{R}^{3}
\end{aligned}
$$

\section{Scheme 18}

\section{1 当量氧化剂氧化的醇与胺的亚胺化反应}

2001 年, Taylor 等 ${ }^{[13]}$ 首先报道了使 $\mathrm{MnO}_{2}$ 为氧化剂 的芐醇与脂肪胺一锅合成亚胺的方法(Eq. 27). 该反应 适用于较活泼的醇类如芐醇、烯丙醇和炔丙醇, 以及碱 性和亲核性较强的脂肪胺. 但是, 该方法需使用过量的 二氧化锰 $(10$ equiv.)、过量的胺、以及 $4 \AA$ 分子篮为脱水 试剂, 使用二氯甲烷为溶剂在回流条件下进行长时间反 应. 该反应中生成的废物比较多, 给产物纯化带来一定 的麻烦.

$$
\begin{gathered}
\mathrm{ArCH}_{2} \mathrm{OH}+\mathrm{RNH}_{2} \underset{1}{\frac{\mathrm{MnO}_{2}(10 \text { equiv. }), 4 \AA \mathrm{MS}}{\mathrm{CH}_{2} \mathrm{Cl}_{2} \text {, reflux, } 24 \sim 48 \mathrm{~h}}} \mathrm{ArCH}=\mathrm{NR} \\
15 \text { examples, } 77 \% \sim 95 \% \text { yields }
\end{gathered}
$$

2003 年, Medvedeva 等 ${ }^{[2]}$ 报道了与上述反应类似的 方法(Eq. 28). 该方法在无溶剂及微波辅助条件下, 使用 5 equiv. 的硅胶负载的 $\mathrm{MnO}_{2}$, 炔丙醇可快速地与各类胺 反应得到相应的炔基亚胺化合物.

$$
\begin{gathered}
\mathrm{R}_{3} \mathrm{E}=\mathrm{RNH}_{2} \frac{\mathrm{MnO}_{2} / \mathrm{SiO}_{2}(5 \text { equiv. } \mathrm{Mn})}{\mathrm{MW}, 1 \sim 4 \mathrm{~min}} \\
\mathrm{R}_{3} \mathrm{E}= \\
\mathrm{ER}_{3}=\mathrm{SiMe}_{3}, \mathrm{GeEt}_{3} ; \mathrm{R}=\text { alkyl, aryl, heteroaryl } \\
8 \text { examples, } 43 \% \sim 96 \% \text { yields }
\end{gathered}
$$

2006 年, Zhdankinc 等 ${ }^{[53]}$ 报道了 $\mathrm{Ru}$ 催化的、二醋酸 碘苯为氧化剂的醇与取代苯肼的分步合成苯腙 18 的反 应(Scheme 19). 该反应首先使用二醋酸碘苯、 $\mathrm{RuCl}_{3}$ 氧 化体系将醇在室温下氧化为醛酮, 再加入苯肼、浓硫酸 等与现场生成的醛酮反应，过滤重结晶可得产物. 该方 法底物适用性较广, 茮醇、杂苠醇、烯丙醇、脂肪醇和 仲醇等都可用于反应，但是该方法需使用重金属 $\mathrm{Ru}$ 催 化剂、当量氧化剂二醋酸碘苯、浓硫酸等试剂, 也产生 不少的废物，因此并不是十分经济绿色的方法.

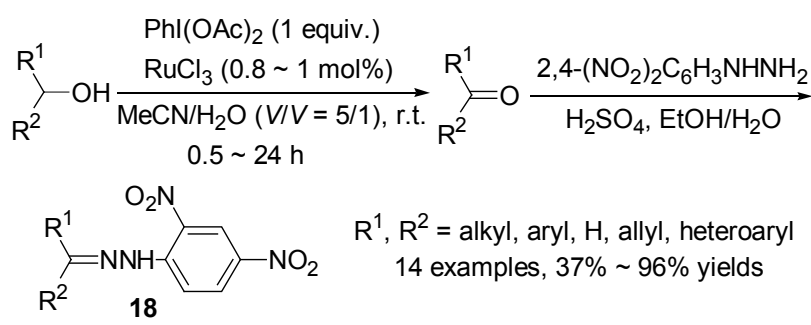

Scheme 19

\section{2 醇与胺在氧气下的有氧氧化缩合反应}

显然，上述使用当量甚至过量氧化剂的方法存在很 多缺点，对传统醛胺缩合的方法改进不是太大. 因而开 发更绿色的、污染小的方法也成为研究者们的目标. 2010 年之后, Milstein 等 ${ }^{[54]}$ 多个课题组相继报道了一些 贵金属催化的醇与胺通过释放出氢气生成亚胺的脱氢 亚胺化反应(Eq. 29).

$$
\begin{aligned}
& \mathrm{R}_{\mathbf{1}}^{1} \underset{\mathrm{OH}}{\widehat{\mathrm{O}}} \underset{\mathbf{2}}{\mathrm{R}^{2} \mathrm{NH}_{2}} \stackrel{\text { cat. Ru, Ir, Os, etc. }}{\underset{\text { up to } 163^{\circ} \mathrm{C}}{\longrightarrow}} \mathrm{R}^{1} \underset{\mathbf{5}}{\stackrel{\mathrm{N}}{\mathrm{N}}} \mathrm{R}^{2}+ \\
& \mathrm{H}_{2} \mathrm{O}+\mathrm{H}_{2}
\end{aligned}
$$

但是, 由于氢气(一种能源)的生成需要消耗能量, 这类方法都需要使用昂贵、不易制备、不稳定的 Ru, Ir, Os 等贵金属和复杂配体的络合物为催化剂、大量的碱、 惰性气体保护以及长时间的高温加热，而且很多反应只 适用于碱性和亲核性较强的脂肪胺, 不适用于碱性和亲 核性较弱的芳胺和杂芳胺. 此外, 在该类放出氢气的还 原性反应体系中, 非常容易发生将亚胺还原为胺的副反 应，导致反应的选择性低、产率低. 因此，用这类方法制 备亚胺并不实用. 相比较而言, 使用氧气或空气为氧化 剂的有氧氧化缩合方法, 由于使用对氧气或空气稳定的 催化剂、不需要惰性气体保护、反应唯一副产物为水, 是 一种更加经济绿色的方法. 目前, 醇与胺的有氧氧化缩 
合反应以氧气为氧化剂的报道为主.

2009 年, Park 等 ${ }^{[29 a]}$ 使用非均相 $\mathrm{Pd}$ 催化剂 $\mathrm{Pd} /$ $\mathrm{AlO}(\mathrm{OH}), 101 \mathrm{kPa}$ 的氧气、 $90{ }^{\circ} \mathrm{C}$ 下, 实现了醇和胺氧化 缩合的亚胺化反应(Eq. 30). 该方法基本只适用于苠醇, 脂肪醇收率非常低( $8 \%$ ), 仲醇不反应。脂肪胺和芳胺都 可以用于反应，普遍收率较高.

$$
\begin{aligned}
& \mathrm{Ar} \widehat{\mathrm{OH}}+\mathrm{RNH}_{2} \frac{\mathrm{Pd} / \mathrm{AlO}(\mathrm{OH})(2 \mathrm{~mol} \%), 101 \mathrm{kPa} \mathrm{O} \text {, heptane }}{90^{\circ} \mathrm{C}, 20 \sim 24 \mathrm{~h}} \\
& \mathrm{Ar} \backslash \mathrm{N}_{-\mathrm{R}}+\mathrm{H}_{2} \mathrm{O} \quad \mathrm{R}=\text { alkyl, aryl } \\
& 11 \text { examples, } 82 \% \sim 98 \% \text { yields }
\end{aligned}
$$

$\mathrm{Cao}$ 等 $^{[55]}$ 在 2009 年也报道, 使用非均相金/着磷灰 石催化剂 $(\mathrm{Au} / \mathrm{HAP})$ 、氧气为氧化剂, 可在较温和的温度 下 $\left(60{ }^{\circ} \mathrm{C}\right)$ 实现醇与胺合成亚胺的反应(Eq. 31). 该反应 适用于苠醇、烯丙醇、脂肪醇、苯胺、脂肪烷胺, 收率 普遍较高. 该方法使用的催化剂合成方法较为复杂, 但 可回收利用. 此外, 该方法也可用于稍大规模的反应 (10 mmol)以及醇与盐酸羟胺的反应用于肟的合成.

$$
\begin{aligned}
& \mathrm{R}^{1} \mathrm{CH}_{2} \mathrm{OH}+\mathrm{R}^{2} \mathrm{NH}_{2} \frac{\text { Au/HAP }(1 \mathrm{~mol} \% \mathrm{Au})}{\mathrm{O}_{2} \text {, toluene, } 60^{\circ} \mathrm{C}, 1 \sim 16 \mathrm{~h}} \\
& \mathrm{R}^{1}-\mathrm{CH}=\mathrm{NR}^{2}+\mathrm{H}_{2} \mathrm{O} \\
& \mathrm{R}^{1}=\text { Aryl, Cinnamyl, } \mathrm{N} \text {-Heptyl; } \mathrm{R}^{2}=\text { Aryl, Alkyl } \\
& \quad 17 \text { examples, } 75 \% \sim 99 \% \text { yields }
\end{aligned}
$$

同年, Mizuno 等 ${ }^{[56]}$ 也报道了氧气下、 $\mathrm{TiO}_{2}$ 负载的 $\mathrm{Ru}(\mathrm{OH})_{x}$ 非均相催化剂催化的醇与胺合成亚胺的反应 (Eq. 32). 该方法适用于苠醇、杂茮醇、脂肪胺、芳胺和 杂芳胺, 但需要在较高的温度下 $\left(100{ }^{\circ} \mathrm{C}\right)$ 进行.

$$
\begin{gathered}
\mathrm{R}^{1} \widehat{\mathrm{OH}}+\mathrm{R}^{2}-\mathrm{NH}_{2} \underset{\begin{array}{c}
\mathrm{Ru}(\mathrm{OH})_{x} / \mathrm{TiO}_{2}(2 \mathrm{~mol} \% \mathrm{Ru}) \\
\text { toluene, } 100^{\circ} \mathrm{C}
\end{array}}{\mathrm{O}_{2}(101 \mathrm{kPa}), 1 \sim 6 \mathrm{~h}} \mathrm{R}^{1} \sim \mathrm{N}^{-} \mathrm{R}^{2} \text { (32) } \\
\mathrm{R}^{1}=\text { aryl, cinnamyl, thiophenol, pyridinyl } \\
\mathrm{R}^{2}=\text { aryl, 2-pyridinyl, tertiary butyl } \\
11 \text { examples, } 62 \% \sim 98 \% \text { yields }
\end{gathered}
$$

2010 年, Riisager 等 ${ }^{[57]}$ 报道了 $\mathrm{TiO}_{2}$ 负载的纳米金催 化剂催化的茮醇与脂肪胺在氧气下的亚胺化反应 (Eq. 33). 研究发现, 升高温度对反应没有明显的作用, 因此 该反应在室温下进行, 但是底物范围很窄, 只适用于茮 醇、烯丙醇和脂肪胺, 而且收率都不高. 仲醇的收率很 低(14\%), 而芳胺的反应只有极少量的产物生成(7\%).

$$
\begin{aligned}
& \mathrm{Au} / \mathrm{TiO}_{2}(0.02 \mathrm{~mol} \%) \\
& \begin{array}{c}
\mathrm{R}^{1} \widehat{\mathrm{OH}}+\mathrm{R}^{2}-\mathrm{NH}_{2} \underset{\begin{array}{c}
\mathrm{CH} \text { OH, r,t. } \sim 65^{\circ} \mathrm{C} \\
\mathrm{O}_{2}(101 \mathrm{kPa}), 24 \mathrm{~h}
\end{array}}{\stackrel{\mathrm{CH}_{3} \mathrm{OK}(10 \mathrm{~mol} \%)}{\longrightarrow}} \mathrm{R}^{1} \mathrm{~N}^{-} \mathrm{R}^{2} \\
\mathrm{R}^{1}=\text { aryl, allyl; } \mathrm{R}^{2}=\text { alkyl } \\
4 \text { examples, } 23 \% \sim 63 \% \text { yields }
\end{array}
\end{aligned}
$$

2011 年, $\mathrm{Yu}$ 等 ${ }^{[58]}$ 报道了氧化铝负载的双金属催化 剂 $\mathrm{Pt}-\mathrm{Sn} / \gamma-\mathrm{Al}_{2} \mathrm{O}_{3}$ 催化的醇与胺在氧气下的有氧亚胺化反 应(Eq. 34). 该方法主要适用于苠醇与芳胺，也可用于少 部分脂肪醇、烯丙醇和仲醇, 产物收率普遍较高, 但是 该反应需要 $138{ }^{\circ} \mathrm{C}$ 的高温.

$$
\begin{aligned}
& \mathrm{Pt}-\mathrm{Sn} / \mathrm{\gamma}-\mathrm{Al}_{2} \mathrm{O}_{3}(0.3 \mathrm{~mol} \% \mathrm{Pt}) \\
& \mathrm{R}^{1} \widehat{\mathrm{OH}}+\mathrm{R}^{2}-\mathrm{NH}_{2} \stackrel{0.1 \mathrm{MPa} \mathrm{O}_{2}, \mathrm{PhEt},}{138{ }^{\circ} \mathrm{C}, 24 \sim 48 \mathrm{~h}}
\end{aligned}
$$

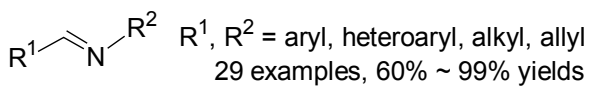

以上使用氧气为氧化剂方法, 比使用过量氧化剂或 者脱氢缩合的方法好，但是都须使用贵金属催化剂，有 些反应还需在高温下进行，实际应用潜力还是有限. 因 此，开发高效低成本的廉价金属催化的反应应用价值更 大. 最近, Zhang 等 ${ }^{[59]}$ 报道了一例使用相对廉价的铜盐 催化的醇与胺的有氧氧化缩合反应(Eq. 35). 该反应使 用水合高氯酸铜为催化剂, 在氧气下、甲苯中 $70{ }^{\circ} \mathrm{C}$ 反 应，可得良好到高收率的亚胺产物. 但是该方法需要使 用过量的胺、大量的碱，反应只适用于苠醇，脂肪族的 伯醇和仲醇基本不反应. 由于使用过量的胺, 会有胺氧 化产物生成，导致反应选择性下降. 研究发现，在该反 应条件下，降低催化剂用量到 $0.005 \mathrm{~mol} \%$, 延长反应时 间到 $72 \mathrm{~h}$, 茮醇与脂肪胺的反应还可以获得高收率的产 物.

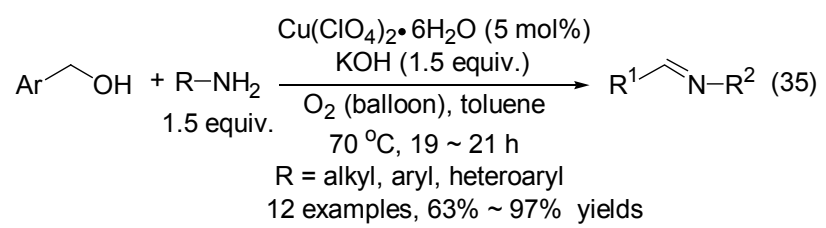

从以上文献报道可以看出, 目前醇与胺有氧氧化合 成亚胺的方法还存在一些不足, 亟需改进: (1)已知方法 往往使用贵金属催化剂, 成本高, 难以普及推广, 因此 开发廉价金属催化剂, 降低反应成本, 使其具备较大规 模制备亚胺的潜力, 值得进一步研究; (2)已知反应往往 需高温加热条件, 否则反应效果不佳 ${ }^{[57]}$, 因此开发高效 的催化剂, 使反应能在更温和的条件下进行、减少能耗, 也非常有意义; (3)以上反应都使用氧气为氧化剂, 但是 高温下使用纯氧存在不少安全隐患，因此改用更经济、 安全、绿色的空气为氧化剂的反应可以在一定程度上降 低成本和解决安全问题, 也非常值得研究; (4)上述方法 很多底物范围有限, 远不如传统的醛胺缩合方法, 因此 开发底物适用范围较广的催化方法, 也是研究中需要考 虑的问题. 
因此, 在醇与胺有氧氧化合成亚胺的研究中, 提出 相应的改进方法, 以解决上述存在的问题, 仍是该研究 的难点和挑战.

\section{3 醇与胺在空气中的有氧氧化缩合反应}

目前, 以空气为氧化剂的亚胺合成方法报道不多, 但是其进展颇为乐观.

2008 年, Suib 等 ${ }^{\left[{ }^{[0]}\right.}$ 报道了八面体 $\mathrm{Mn}$ 分子篎 (K-OMS-2)催化的醇与胺氧化缩合制备亚胺的反应(Eq. 36). 该反应可使用空气为氧化剂, 但是需使用过量的胺 和 $110{ }^{\circ} \mathrm{C}$ 的高温. 该方法底物范围较窄, 主要适用于苄 醇和杂苠醇, 脂肪醇和仲醇的收率很低(12\% 44\%).

$$
\begin{aligned}
& \mathrm{R}^{1} \widehat{\mathrm{OH}}+\underset{2 \text { equiv. }}{\mathrm{R}^{2}-\mathrm{NH}_{2}} \underset{\text { air, toluene, } 110{ }^{\circ} \mathrm{C}}{\stackrel{\text { cat. K-OMS-2 }}{12 \sim 24 \mathrm{~h}}} \mathrm{R}^{1} \widehat{\mathrm{N}^{-}} \mathrm{R}^{2} \\
& \mathrm{R}^{1}=\text { aryl, heteroaryl, cinnamyl, alkyl; } \mathrm{R}^{2}=\mathrm{Ph}, n-\mathrm{Bu} \\
& 16 \text { examples, } 12 \% \sim 99 \% \text { yields }
\end{aligned}
$$

2011 年, $\mathrm{Xu}$ 等 ${ }^{[22,26]}$ 在金属催化的有氧 $N$-烷基化反 应的基础上，报道了空气下 Pd 催化的醇与胺的有氧氧 化的亚胺合成法(Eq. 37 ${ }^{[41]}$. 通过催化剂和反应条件的 优化，该反应使用低用量的商业化的催化剂 $\mathrm{Pd}(\mathrm{OAc})_{2}$ $(1 \mathrm{~mol} \% \mathrm{Pd})$ 、稳定的三乙胺为配体、 TEMPO 为共氧化 剂, 在催化量的碱的存在下, 无需溶剂, 可在室温空气 的温和条件下从醇和胺出发制备亚胺. 该方法主要适用 于芐醇、杂芐醇、烯丙醇和脂肪胺, 可得中等到高收率 的产物. 芳胺的收率比较低, 但是通过增加碱的用量可 提高产率. 虽然该方法需使用过量的醇, 反应时间也较 长, 却是第一例实现的室温下空气为氧化剂的醇与胺的 有氧氧化缩合反应.

$$
\begin{aligned}
& \mathrm{R}^{1} \widehat{\mathrm{OH}}+\mathrm{R}^{2} \mathrm{NH}_{2} \frac{\begin{array}{c}
\mathrm{Pd}(\mathrm{OAc})_{2}(1 \mathrm{~mol} \%) \\
\frac{\mathrm{Et}_{3} \mathrm{~N}(15 \mathrm{~mol} \%)}{t-\mathrm{BuOK}(20 \mathrm{~mol} \%)} \\
\text { air, neat, r.t., } 3 \mathrm{~d}
\end{array}}{\mathrm{R}^{1}} \mathrm{R}^{1} \widehat{\mathrm{N}}^{-} \mathrm{R}^{2} \\
& \mathrm{R}^{1}=\text { aryl, heteroaryl, allyl; } \mathrm{R}^{2}=\text { alkyl, aryl } \\
& 32 \text { examples, } 21 \% \sim 99 \% \text { yields }
\end{aligned}
$$

其后, $\mathrm{Xu}$ 等 ${ }^{[42]}$ 继续开发了室温空气下 $\mathrm{Cu}$ 催化的醇 与胺有氧氧化制备亚胺的方法(Scheme 20). 该反应使用 更经济易得的 $\mathrm{Cu}$ 催化剂, 但是发现该催化剂活性更高, 大大改善了之前报道的钯催化 ${ }^{[41]}$ 和铜催化方法 ${ }^{[59]}$ 缺点 和不足, 使得反应在温和条件下可以更加高效和快速地 进行. 如 Scheme 20 所示, 该反应基本使用 $1: 1$ 的醇与 胺, 在室温空气敞口条件下, 无需加入碱和脱水试剂, 使用 $1 \mathrm{~mol} \%$ 的 $\mathrm{Cu}$ 催化剂, 在中性条件下即可快速地在 6 24 h 内完成反应, 得到中等到高收率的产物. 该方法 底物适用范围更广, 芐醇、杂芐醇、烯丙醇、炔丙醇、 脂肪醇都可以用于反应; 脂肪胺的反应都可得到高产率 的产物, 而弱亲核性的芳胺和杂芳胺的反应效果也比以
上报道的其他方法好很多, 可得到中等以上收率的产 物. 研究发现, $\mathrm{Cu}$ 催化剂对醛与胺的缩合起促进作用, 可以解释芳胺和杂芳胺在 $\mathrm{Cu}$ 催化方法中较好的反应效 果, 也显示了使用 $\mathrm{Cu}$ 催化剂的优势. 此外, 手性胺参与 的反应中，未发现手性原料和产物的消旋现象，可得到 光学纯的手性亚胺产物. 由于温和的反应条件, 该反应 中没有观察到胺自身氧化的副产物和其他可能的副产 物, 因此亚胺产物的选择性也很高, 分离收率也较高. 此外, $100 \mathrm{mmol}$ 级的放大实验可得到 $89 \%$ 收率的产物, 也说明该方法具备用于大量亚胺制备的潜力.

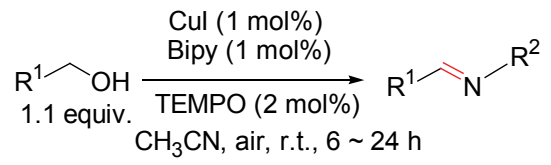

$$
\begin{aligned}
& \mathrm{R}^{1} \overbrace{\mathrm{OH}}+\mathrm{H}_{2} \mathrm{~N}-\mathrm{R}^{2} \stackrel{\begin{array}{l}
\text { Cul }(1 \mathrm{~mol} \%) \\
\text { Bipy }(1 \mathrm{~mol} \%)
\end{array}}{\operatorname{TEMPO}(2 \mathrm{~mol} \%)} \\
& 1.1 \text { equiv. } \quad \mathrm{CH}_{3} \mathrm{CN} \text {, air, r.t., } 6 \sim 24 \mathrm{~h} \\
& \mathrm{R}^{1} \curvearrowright \mathrm{N}^{-\mathrm{R}^{2}}+\mathrm{H}_{2} \mathrm{O}
\end{aligned}
$$

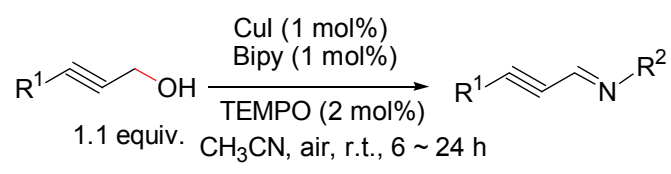

$$
\begin{aligned}
& \mathrm{R}^{1}, \mathrm{R}^{2}=\text { alkyl, aryl, heteroaryl } \\
& 46 \text { examples, up to } 99 \% \text { yield }
\end{aligned}
$$

\section{Scheme 20}

对比上述报道的其他的醇与胺有氧氧化缩合合成 亚胺的方法, $\mathrm{Xu}$ 等这一 $\mathrm{Cu}$ 催化的方法基本上解决了原 先研究中存在的催化剂的来源和价格、反应的条件和温 度、空气的有效利用和底物范围窄等方面存在的问题, 因此可以说是目前所知的最好的醇与胺有氧氧化反应 合成亚胺的方法.

\section{4 醇与甲基酮的有氧氧化缩合反应}

与上述醇与胺的有氧氧化合成亚胺的反应类似, 通 过反应条件的控制和优化，醇与甲基酮的有氧 $C$-烷基化 反应可进一步运用于有氧氧化缩合反应合成 $\alpha, \beta$-不饱和 酮. 2005 年, Park 等 ${ }^{[61]}$ 在研究甲基酮的 $C$-烷基化反应时 发现, 使用同样的非均相 $\mathrm{Pd}$ 催化剂 $\mathrm{Pd} / \mathrm{AlO}(\mathrm{OH})$, 将反 应在氧气下进行, 即可拿到良好收率的 $\alpha, \beta$-不饱和酮产 物(Eq. 38). 该反应报道的底物范围有限, 且需要大量的 碱和高温加热的反应条件.

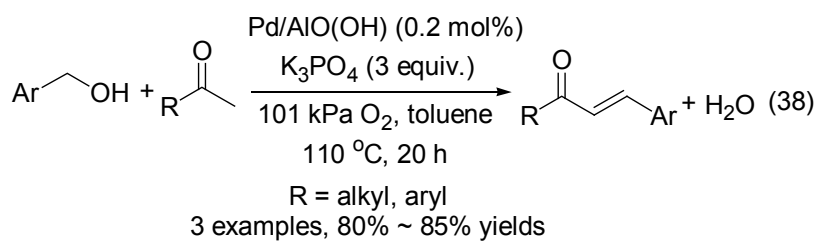


2009 年, Park 等 ${ }^{[62]}$ 对上述方法进行了一些改进, 使 用可回收的纳米 $\mathrm{Au}$ 催化剂 $\mathrm{Au} / \mathrm{AlO}(\mathrm{OH})$ 后, 可在室温下 实现 $\alpha, \beta$-不饱和酮的合成(Eq. 39). 但是该方法还是需使 用过量的醇、大量的碱. 该反应底物范围与上述 $\mathrm{Pd}$ 催化 方法类似，只适用于苠醇，脂肪伯醇的效果不好.

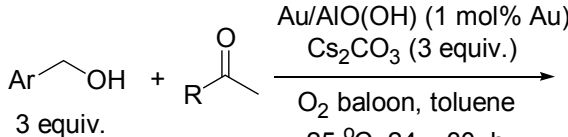

$$
\begin{aligned}
& 25^{\circ} \mathrm{C}, 24 \sim 30 \mathrm{~h} \\
& \mathrm{R} \stackrel{\mathrm{O}}{\mathrm{Ar}}+\mathrm{H}_{2} \mathrm{O} \quad \mathrm{R}=\mathrm{alkyl} \text {, aryl } \\
& 7 \text { examples, } 70 \% \sim 90 \% \text { yields }
\end{aligned}
$$

在上述 $\mathrm{Cu}$ 催化 $C$-烷基化反应 ${ }^{[48]}$ 和亚胺合成方法 ${ }^{[42]}$ 的基础上, $\mathrm{Xu}$ 等 ${ }^{[63]}$ 后来也开发了 $\mathrm{Cu}$ 催化的醇与甲基酮 的有氧氧化反应制备 $\alpha, \beta$-不饱和酮的方法(Eq. 40). 该反 应使用类似的 $\mathrm{Cu}$ 催化剂体系, 使用 1 equiv. 的碱即可在 空气、室温的温和条件下实现 $\alpha, \beta$-不饱和酮的合成. 由 于温和的反应条件, 该方法的底物范围较广, 适用于一 系列伯醇和取代酮底物, 也有望成为一种普遍适用的 $\alpha, \beta$-不饱和酮的绿色合成法.

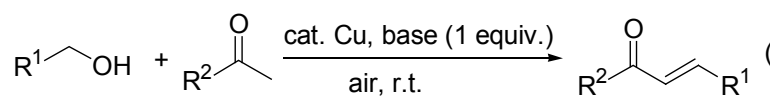

\section{3 结论与展望}

近年来, 绿色化学和绿色合成技术的研究开发越来 越受到了人们的重视. 与相关的卤代烃或者醛酮等毒性 较大的化合物相比, 醇是一种相对绿色的合成试 剂 $^{[9,11,12 \mathrm{a} \sim 12 \mathrm{~b}]}$. 因此, 直接使用醇为反应原料的合成和应 用研究也引起了各个领域的普遍关注. 例如, 2005 年, 美国化学会(ACS)的绿色化学协会(GCI)和全球多家制 药公司联合发起了关于制药行业的圆桌协会(ACS GCI Pharmaceutical Roundtable), 旨在倡导和推动绿色化学
和绿色工程在药物研发和制药工业中的应用研究. 在该 协会支持的诸多研究方向中就包括醇的活化和以醇为 直接底物的绿色合成方法研究.

由于醇差基不活泼，因此醇的活化方法研究，特别 是温和高效且绿色的活化方法，对醇类化合物在有机合 成及药物和复杂分子合成中的应用都具有很好的借鉴 和指导作用. 近年来, 基于醇活化的醇与胺和酰胺等化 合物在有氧条件下的脱水反应研究取得了一定的进展, 因此本文总结了直接使用醇为合成试剂、通过氧化的活 化方式将醇转化为更活泼的醛酮化合物, 继而参与一锅 多步反应合成胺和酰胺衍生物、亚胺化合物、多取代醇 和酮、以及 $\alpha, \beta$-不饱和酮等有用的化合物的一系列相对 绿色的合成方法(Scheme 21), 对相关反应的机理研究也 进行了一定的讨论.

总结这些反应，并与相关的醇的脱氢活化(Scheme 22 , 方法 $\mathrm{A} \sim \mathrm{C})^{[9,11]}$ 和胺类与醚类化合物的 $\mathrm{CDC}$ 活化 ${ }^{[36]}$ 相比较, 可以看出, 氧化剂对醇的氧化也是一种可取的 醇的活化方式(Scheme 22, 方法 D 和 E). 其中，过渡金 属催化的氧气或空气下的醇氧化(方法 $\mathrm{E}$ ), 由于不使用 产生大量废物和污染的常规氧化剂, 副产物为水, 还可 以通过水的生成降低醇活化的难度、促进活化过程, 因 此是一种更优势和更绿色的活化方式. 遗憾的是，虽然 有不少研究报道了氧化剂作用下的多步 $N$-烷基化反应 (第 1.1 节)、有氧氧化的亚胺化反应(第 $2.1 \sim 2.3$ 节)和有 氧氧化的烯酮化反应(第 2.4 节), 甚至过渡金属催化的 氧气或空气下的醇氧化反应 ${ }^{[23,37]}$ 也已经是广为人知和 普遍应用的研究，在胺、酰胺、仲醇和甲基酮等化合物 的脱水烷基化反应研究中(第 1.2, 1.3 节), 该方法作为一 种新的醇的活化方式尚未完全得到该领域研究者的理 解和认同.

此外，目前报道的过渡金属催化的醇与胺、酰胺、

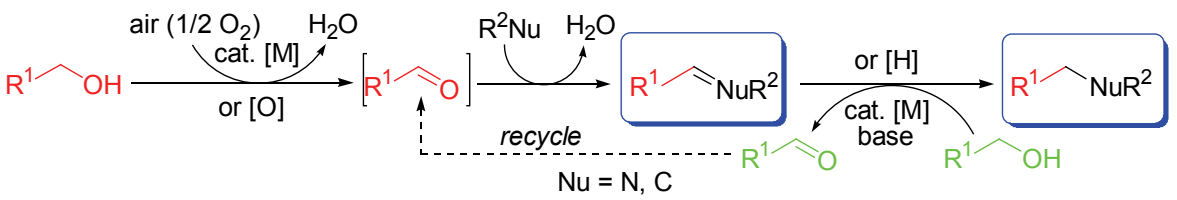

Scheme 21

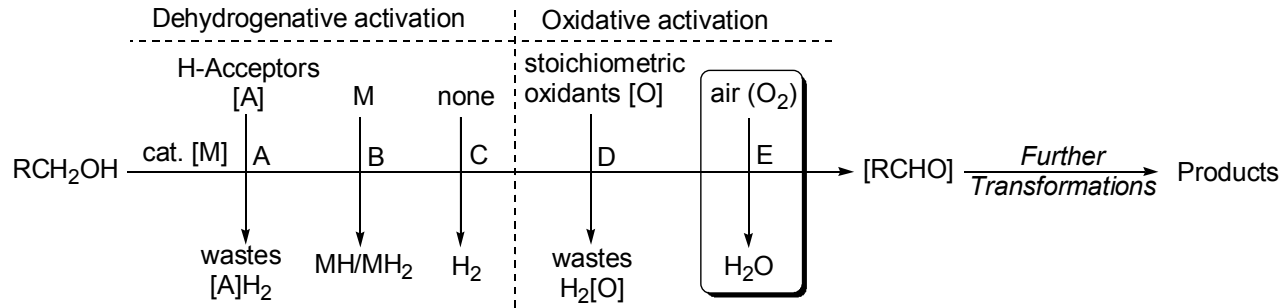

Scheme 22 
仲醇和甲基酮的有氧脱水 $\mathrm{C}-\mathrm{N}, \mathrm{C}=\mathrm{N}, \mathrm{C}-\mathrm{C}$ 和 $\mathrm{C}=\mathrm{C}$ 键形成的反应，主要使用了各种贵重金属催化剂，价格 昂贵、难以扩大反应规模和进一步地推广应用. 因此, 开发经济易得、环境更加友好和亲生物的金属催化剂体 系(例如铜和铁等), 甚至无过渡金属催化的新方法, 进 一步改进和拓展现有催化方法和反应的底物适用范围, 以发展更多的绿色合成方法, 是今后研究的重要方向. 由于目前有氧烷基化反应研究中, 反应的机理尚存不少 争议, 通过更有力的机理实验、更深入的机理探讨、甚 至理论计算等方面的研究工作进一步研究和确证反应 的机理, 在今后的研究中显得非常的必要和重要, 也将 有助于该领域研究成果的进一步拓展和更广泛的应用.

\section{References}

[1] (a) Brooks, G. T.; Roberts, T. R. Pesticide Chemistry and Bioscience, Royal Society of Chemistry, Cambridge, U.K., 1999.

(b) McGuire, J. L. Pharmaceuticals: Classes, Therapeutic Agents, Areas of Application, Vols. 1 4, Wiley-VCH, Weinheim, Germany, 2000 .

(c) Hili, R.; Yudin, A. K. Nat. Chem. Biol. 2006, 2, 284.

(d) Cox, E. D.; Cook, J. M. Chem. Rev. 1995, 95, 1797.

(e) Humphrey, J. M.; Chamberlin, A. R. Chem. Rev. 1997, 97, 2243.

(f) Hansch, C.; Sammes, P. G.; Taylor, J. B. Comprehensive Medicinal Chemistry, Vol. 2, Pergamon Press, Oxford, 1990, Chapter 7.1.

(g) Connor, E. E. Prim. Care Update Ob/Gyns 1998, 5, 32.

(h) Kleemann, A.; Engel, J.; Kutscher, B.; Reichert, D. Pharmaceutical Substances, Synthesis, Patents, Applications, Thieme, Stuggart, 1999.

(i) Park, S.-Y.; Fung, P.; Nishimura, N.; Jensen, D. R.; Fujii, H.; Zhao, Y.; Lumba, S.; Santiago, J.; Rodrigues, A.; Chow, T. F.; Alfred, S. E.; Bonetta, D.; Finkelstein, R.; Provart, N. J.; Desveaux, D.; Rodriguez, P. L.; McCourt, P.; Zhu, J.-K.; Schroeder, J. I.; Volkman, B. F.; Cutler, S. R. Science 2009, 324, 1068.

[2] (a) Bloch, R. Chem. Rev. 1998, 98, 1407.

(b) Yet, L. Angew. Chem., Int. Ed. 2001, 40, 875.

(c) Ellman, J. A.; Owens, T. D.; Tang, T. P. Acc. Chem. Res. 2002, 35,984 .

(d) Taggi, A. E.; Hafez, A. M.; Lectka, T. Acc. Chem. Res. 2003, 36,10 .

(e) Córdova, A. Acc. Chem. Res. 2004, 37, 102.

(f) Ma, J.-A. Chem. Soc. Rev. 2006, 35, 630.

(g) Dömling, A. Chem. Rev. 2006, 106, 17.

(h) Erkkilä, A.; Majander, I.; Pihko, P. M. Chem. Rev. 2007, 107, 5416 .

(i) Gawronski, J.; Wascinska, N.; Gajewy, J. Chem. Rev. 2008, 108, 5227.

(j) Yamada, K.-I.; Tomioka, K. Chem. Rev. 2008, 108, 2874.

(k) Merino, P.; Marqués-López, E.; Herrera, R. P. Adv. Synth. Catal. 2008, 350, 1195.

(1) Ordóñez, M.; Rojas-Cabrera, H.; Cativiela, C. Tetrahedron 2009, $65,17$.

(m) Martin, S. F. Pure Appl. Chem. 2009, 81, 195.

(n) de Armas, P.; Tejedor, D.; Garía-Tellado, F. Angew. Chem., Int. Ed. 2010, 49, 1013.

(o) Li, C.-J. Acc. Chem. Res. 2010, 43, 581. (p) Akhmetova, V. R.; Khabibullina, G. R.; Rakhimova, E. B.; Vagapov, R. A.; Khairullina, R. R.; Niatshina, Z. T.; Murzakova, N. N. Mol. Diversity 2010, 14, 463.

(q) Yin, B.; Zhang, Y.; Xu, L.-W. Synthesis 2010, 3583.

(r) Kobayashi, S.; Mori, Y.; Fossey, J. S.; Salter, M. M. Chem. Rev. 2011, 111, 2626.

(s) Xie, J.-H.; Zhu, S.-F.; Zhou, Q.-L. Chem. Rev. 2011, 111, 1713.

(t) Adrio, J.; Carretero, J. C. Chem. Commun. 2011, 47, 6784.

(u) Marques, C. S.; Burke, A. J. ChemCatChem 2011, 3, 635.

(v) Ramadhar, T. R.; Batey, R. A. Synthesis 2011, 1321.

(w) Nielsen, M.; Worgull, D.; Zweifel, T.; Gschwend, B.; Bertelsen, S.; Jøgensen, K. A. Chem. Commun. 2011, 47, 632.

[3] (a) Hartwig, J. F. Acc. Chem. Res. 2008, 41, 1534.

(b) Surry, D. S.; Buchwald, S. L. Angew. Chem., Int. Ed. 2008, 47, 6338.

(c) Evano, G.; Blanchard, N.; Toumi, M. Chem. Rev. 2008, 108, 3054.

[4] (a) Tripathi, R. P.; Verma, S. S.; Pandey, J.; Tiwari, V. K. Curr. Org. Chem. 2008, 12, 1093.

(b) Burkhardt, E. R.; Karl Matos, K. Chem. Rev. 2006, 106, 2617.

(c) Baxter, E. W.; Reitz, A. B. In Organic Reactions, Vol. 59, Ed.: Overman, L. E., Wiley, Singapore, 2002.

[5] (a) Müller, T. E.; Hultzsch, K. C.; Yus, M.; Foubelo, F.; Tada, M. Chem. Rev. 2008, 108, 3795.

(b) Severin, R.; Doye, S. Chem. Soc. Rev. 2007, 36, 1407.

(c) Müller, T. E.; Beller, M. Chem. Rev. 1998, 98, 675.

[6] (a) Veige, A. S. Polyhedron 2008, 27, 3177.

(b) Beccalli, E. M.; Broggini, G.; Martinelli, M.; Sottocornola, S. Chem. Rev. 2007, 107, 5318.

(c) Evano, G.; Coste, A.; Jouvin, K. Angew. Chem., Int. Ed. 2010 , $49,2840$.

(d) DeKorver, K. A.; Li, H.; Lohse, A. G.; Hayashi, R.; Lu, Z.; Zhang, Y.; Hsung, R. P. Chem. Rev. 2010, 110, 5064.

[7] (a) Salvatore, R. N.; Yoon, C. H.; Jung, K. W. Tetrahedron 2001, 57,7785 .

(b) Chiappe, C.; Pieraccini, D. Green Chem. 2003, 5, 193.

[8] Nef, J. U. Liebigs Ann. Chem. 1901, 318, 137.

[9] Guillena, G.; Ramón, D. J.; Yus, M. Chem. Rev. 2010, 110, 1611.

[10] (a) Grigg, R.; Mitchell, T. R. B.; Sutthivaiyakit, S.; Tongpenyai, N. J. Chem. Soc., Chem. Commun. 1981, 611.

(b) Watanabe, Y.; Tsuji, Y.; Ohsugi, Y. Tetrahedron Lett. 1981, 22, 2667.

(c) Maruhashi, S.-I.; Kondo, K.; Hakata, T. Tetrahedron Lett. 1982, 23, 229 .

[11] (a) Watson, A. J. A.; Williams, J. M. J. Science 2010, 329, 635.

(b) Nixon, T. D.; Whittlesey, M. K.; Williams, J. M. J. Dalton Trans. 2009, 753.

(c) Hamid, M. H. S. A.; Slatford, P. A.; Williams, J. M. J. $A d v$. Synth. Catal. 2007, 349, 1555.

(d) Suzuki, T. Chem. Rev. 2011, 111, 1825.

(e) Bähn, S.; Imm, S.; Neubert, L.; Zhang, M.; Neumann, H.; Beller, M. ChemCatChem 2011, 3, 1853.

(f) Crabtree, R. H. Organometallics 2011, 30, 17.

(g) Dobereiner, G. E.; Crabtree, R. H. Chem. Rev. 2010, 110, 681.

(h) Guillena, G.; Ramón, D. J.; Yus, M. Angew. Chem., Int. Ed. 2007, 46, 2358.

(i) Fujita, K.-I.; Yamaguchi, R. Synlett 2005, 560.

[12] (a) Emer, E.; Sinisi, R.; Capdevila, M. G.; Petruzziello, D.; De Vincentiis, F.; Cozzi, P. G. Eur. J. Org. Chem. 2011, 647 and references cited therein.

(b) Guérinot, A.; Reymond, S.; Cossy, J. Eur. J. Org. Chem. 2011, 647. 
(c) Qin, H.; Yamagiwa, N.; Matsunaga, S.; Shibasaki, M. Angew. Chem., Int. Ed. 2007, 46, 409.

(d) Zhan, Z.-P.; Yang, W.-Z.; Yang, R.-F.; Yu, J.-L.; Li, J.-P.; Liu, H.-J. Chem. Commun. 2006, 3352.

(e) Terrasson, V.; Marque, S.; Georgy, M.; Campagne, J.-M.; Prim, D. Adv. Synth. Catal. 2006, 348, 2063.

(f) Zhan, Z.-P.; Yu, J.-L.; Liu, H.-J.; Cui, Y.-Y.; Yang, R.-F.; Yang, W.-Z.; Li, J.-P. J. Org. Chem. 2006, 71, 8298.

(g) Zhao, Y.; Foo, S. W.; Saito, S. Angew. Chem., Int. Ed. 2011, 50, 3006 .

(h) Tsai, C.-Y.; Sung, R.; Zhuang, B.-R.; Sung, K. Tetrahedron 2010, 66, 6869.

(i) Sreedhar, B.; Reddy, P. S.; Reddy, M. M.; Neelima B.; Arundhathi, R. Tetrahedron Lett. 2007, 48, 8174.

(j) Das, B.; Reddy, P. R.; Sudhakar, C.; Lingaiah, M. Tetrahedron Lett. 2011, 52, 3521.

(k) Ozawa, F.; Okamoto, H.; Kawagishi, S.; Yamamoto, S.; Minami, T.; Yoshifuji, M. J. Am. Chem. Soc. 2002, 124, 10968.

(1) Ohshima, T.; Miyamoto, Y.; Ipposhi, J.; Nakahara, Y.; Utsunomiya, M.; Mashima, K. J. Am. Chem. Soc. 2009, 131, 14317.

(m) Utsunomiya, M.; Miyamoto, Y.; Ipposhi, J.; Ohshima, T.; Mashima, K. Org. Lett. 2007, 9, 3371.

(n) Tao, Y.; Wang, B.; Wang, B.; Qu, L.; Qu, J. Org. Lett. 2010, 12, 2726.

(o) Roggen, M.; Carreira, E. M. J. Am. Chem. Soc. 2010, 132, 11917.

[13] Blackburn, L.; Taylor, R. J. K. Org. Lett. 2001, 3, 1637.

[14] Kanno, H.; Taylor, R. J. K. Tetrahedron Lett. 2002, 43, 7337.

[15] Guerin, C.; Bellosta, V.; Guillamot, G.; Cossy, J. Org. Lett. 2011, 13,3534 .

[16] Guerin, C.; Bellosta, V.; Guillamot, G.; Cossy, J. Eur. J. Org. Chem. 2012, 15, 2990.

[17] Likhar, P. R.; Arundhathi, R.; Kantam, M. L. Eur. J. Org. Chem. 2009, 5383.

[18] Shi, F.; Tse, M. K.; Cui, X.; Goerdes, D.; Michalik, D.; Thurow, K.; Deng, Y.; Beller, M. Angew. Chem., Int. Ed. 2009, 48, 5912.

[19] (a) Martínez-Asencio, A.; Ramón, D. J.; Yus, M. Tetrahedron Lett. 2010, 51, 325 .

(b) Martínez-Asencio, A.; Ramón, D. J.; Yus, M. Tetrahedron 2011, 67, 3140 .

[20] Cui, X.; Shi, F.; Tse, M. K.; Goerdes, D.; Thurow, K.; Beller, M.; Deng, Y. Adv. Synth. Catal. 2009, 351, 2949.

[21] Feng, S. L.; Liu, C. Z.; Li, Q.; Yu, X. C.; Xu, Q. Chin. Chem. Lett. 2011, 22, 1021.

[22] Liu, C.; Liao, S.; Li, Q.; Feng, S.; Sun, Q.; Yu, X.; Xu, Q. J. Org. Chem. 2011, 76, 5759 and references cited therein.

[23] (a) Sheldon, R. A.; Arends, I. W. C. E.; Brink, G.-J. T.; Dijksman, A. Acc. Chem. Res. 2002, 35, 774 .

(b) Gligorich, K. M.; Sigman, M. S. Chem. Commun. 2009, 3854.

(c) Stahl, S. S. Angew. Chem., Int. Ed. 2004, 43, 3400.

(d) Naota, T.; Takaya, H.; Murahashi, S.-I. Chem. Rev. 1998, 98 , 2599.

(e) Muzart, J. Tetrahedron 2003, 59, 5789.

(f) Arita, S.; Koike, T.; Kayaki, Y.; Ikariya, T. Angew. Chem., Int. Ed. 2008, 47, 2447.

(g) Zhang, J.; Li, S.; Fu, X.; Wayland, B. B. Dalton Trans. 2009, 3661 .

(h) Izumi, A.; Obora, Y.; Sakaguchi, S.; Ishii, Y. Tetrahedron Lett. 2006, 47, 9199.

[24] (a) Chohan, Z. H.; Shad, H. A.; Nasim, F.-H. Appl. Organomet. Chem. 2009, 23, 319 and references therein.

(b) Cejudo-Marín, R.; Alzuet, G.; Ferrer, S.; Borrás, J. Inorg. Chem.
2004, 43, 6805 .

(c) Zhang, T.; Wang, W.; Gu, X.; Shi, M. Organometallics 2008, 27, 753 .

(d) Liang, J.; Lipscomb, W. N. Biochemistry 1989, 28, 9724.

(e) Evelhoch, J. L. E.; Bocian, D. F.; Sudmeier, J. L. Biochemistry 1981, 20, 4951.

(f) Dimroth, J.; Keilitz, J.; Schedler, U.; Schomäcker, R.; Haag, R. Adv. Synth. Catal. 2010, 352, 2497.

[25] (a) Watanabe, Y.; Morisaki, Y.; Kondo, T.; Mitsudo, T. J. Org. Chem. 1996, 61, 4214.

(b) Watanabe, Y.; Tsuji, Y.; Ige, H.; Ohsugi, Y.; Ohta, T. J. Org. Chem. 1984, 49, 3359.

(c) Fujita, K.-I.; Enkoi, Y.; Yamaguchi, R. Tetrahedron 2008, 64 , 1943.

[26] Yu, X.; Jiang, L.; Li, Q.; Xie, Y. ; Xu, Q. Chin. J. Chem. 2012, 30, 2322.

[27] (a) Layer, R. W. Chem. Rev. 1963, 63, 489.

(b) Sprung, M. M. Chem. Rev. 1940, 26, 297.

(c) Patai, S. The Chemistry of the Carbon-Nitrogen Double Bond (Chemistry of Functional Goups), Wiley-Interscience, New York, 1970.

(d) Adams, J. P. J. Chem. Soc., Perkin Trans. 1 2000, 125.

[28] (a) Gladiali, S.; Alberico, E. Chem. Soc. Rev. 2006, 35, 226.

(b) Samec, J. S. M.; B ckvall, J.-E.; Andersson, P. G.; Brandt, P. Chem. Soc. Rev. 2006, 35, 237.

(c) Saluzzo, C.; Lemaire, M. Adv. Synth. Catal. 2002, 344, 915.

(d) Noyori, R.; Hashiguchi, S. Acc. Chem. Res. 1997, 30, 97.

(e) Krische, M. J.; Sun, Y. Special issue on hydrogenation and transfer hydrogenation. Acc. Chem. Res. 2007, 40, issue 12.

[29] (a) Kwon, M. S.; Kim, S.; Park, S.; Bosco, W.; Chidrala, R. K.; Park, J. J. Org. Chem. 2009, 74, 2877.

(b) Corma, A.; Ródenas, R.; Sabater, M. J. Chem. Eur. J. 2010, 16, 254.

(c) Zhang, Y.; Qi, X.; Cui, X.; Shi, F.; Deng, Y. Tetrahedron Lett. 2011, 52, 1334.

[30] Martínez-Asencio, A.; Yus, M.; Ramón, D. J. Synthesis 2011, 3730.

[31] Kawahara, R.; Fujita, K.-I.; Yamaguchi, R. Adv. Synth. Catal. 2011, $353,1161$.

[32] Ohta, H.; Yuyama, Y.; Uozumi, Y.; Yamada, Y. M. A. Org. Lett. 2011, 13, 3892.

[33] Yu, X.; Liu, C.; Jiang, L.; Xu, Q. Org. Lett. 2011, 13, 6184.

[34] (a) Gonzalez-Arellano, C.; Yoshida, K.; Luque, L.; Gai, P. L. Green Chem. 2010 12, 1281.

(b) Martínez, M.; Ramón, D. J.; Yus, M. Org. Biomol. Chem. 2009, 7, 2176.

(c) Valotl, F.; Fachel, F.; Jacquot, R.; Spagnol, M.; Lemairel, M. Tetrahedron Lett. 1999, 40, 3689.

[35] (a) Shi, F.; Tse, M. K.; Zhou, S.; Pohl, M. M.; Radnik, J.; Hübner, S.; J hnisch, K.; Brückner, A.; Beller, M. J. Am. Chem. Soc. 2009, 131,1775 .

(b) Cano, R.; Ramón, D. J.; Yus, M. J. Org. Chem. 2011, 76, 5547.

(c) Yamaguchi, K.; He, J.; Oishi, T.; Mizuno, N. Chem. Eur. J. 2010, 16, 7199 .

(d) He, J.; Kim, J. W.; Yamaguchi, K.; Mizuno, N. Angew. Chem., Int. Ed. 2009, 48, 9888.

(e) Cui, X.; Zhang, Y.; Shi, F.; Deng, Y. Chem. Eur. J. 2011, 17, 1021.

(f) He, L.; Lou, X.-B.; Ni, J.; Liu, Y.-M.; Cao, Y.; He, H.-Y.; Fan, K.-N. Chem. Eur. J. 2010, 16, 13965.

[36] (a) Li, C.-J.; Li, Z. Pure Appl. Chem. 2006, 78, 935.

(b) Li, C.-J. Acc. Chem. Res. 2009, 42, 335.

(c) Scheuermann, C. J. Chem. Asian J. 2010, 5, 436. 
(d) Li, Z.; Bohle, S.; Li, C.-J. Proc. Natl. Acad. Sci. U. S. A. 2006, $103,8928$.

[37] (a) Semmelhack, M. F.; Schmid, C. R.; Cortes, D. A.; Chou, C. S. J. Am. Chem. Soc. 1984, 106, 3374.

(b) Markó, I. E.; Giles, P. R.; Tsukazaki, M.; Brown, S. M.; Urch, C. J. Science 1996, 274, 2044.

(c) Markó, I. E.; Gautier, A.; Dumeunier, R.; Doda, K.; Philippart, F.; Brown, S. M.; Urch, C. J. Angew. Chem., Int. Ed. 2004, 43, 1588 .

(d) Gamez, P.; Arends, I. W. C. E.; Reedijk, J.; Sheldon, R. A. Chem. Commun. 2003, 2414.

(d) Hoover, J. M.; Stahl, S. S. J. Am. Chem. Soc. 2011, 133, 16901.

[38] (a) Wendlandt, A. E.; Suess, A. M.; Stahl, S. S. Angew. Chem., Int. Ed. 2011, 50, 11062.

(b) Liu, C.; Zhang, H.; Shi, W.; Lei, A. Chem. Rev. 2011, 111, 1780 .

(c) Klussmann, M.; Sureshkumar, D. Synthesis 2011, 353.

(d) Zhao, Z.; Peng, F. Angew. Chem., Int. Ed. 2010, 49, 9566.

(e) Stefani, H. A.; Guarezemini, A. S.; Cella, R. Tetrahedron 2010, 66, 7871 .

[39] Li, Q.; Fan, S.; Sun, Q.; Tian, H.; Yu, X.; Xu, Q. Org. Biomol. Chem. 2012, 10, 2966 and references cited therein.

[40] (a) Deutsch, C.; Krause, N.; Lipshutz, B. H. Chem. Rev. 2008, 108, 2916.

(b) Rendler, S.; Oestreich, M. Angew. Chem., Int. Ed. 2007, 46, 498.

(c) Lipshutz, B. H. Synlett 2009, 509.

[41] Jiang, L.; Jin, L.; Tian, H.; Yuan, X.; Yu, X.; Xu, Q. Chem. Commun. 2011, 47, 10833.

[42] Tian, H.; Yu, X.; Li, Q.; Wang, J.; Xu, Q. Adv. Synth. Catal. 2012, 354, 2671.

[43] Yamada, Y. M. A.; Uozumi, Y. Org. Lett. 2006, 8, 1375.

[44] Yamada, Y. M. A.; Uozumi, Y. Tetrahedron 2007, 63, 8492.

[45] Allen, L. J.; Crabtree, R. H. Green Chem. 2010, 12, 1362.

[46] (a) Thomé, I.; Nijs, A.; Bolm, C. Chem. Soc. Rev. 2012, 41, 979.

(b) Leadbeater, N. E. Nat. Chem. 2010, 2, 913.

(c) Buchwald, S. L.; Bolm, C. Angew. Chem., Int. Ed. 2009, 48, 5586.

[47] Tang, G.; Cheng, C.-H. Adv. Synth. Catal. 2011, 353, 1918.

[48] Liao, S.; Yu, K.; Li, Q.; Tian, H.; Zhang, Z.; Yu, X.; Xu, Q. Org. Biomol. Chem. 2012, 10, 2973 and references cited therein.
[49] (a) Palacios, F.; Alonso, C.; Aparicio, D.; Rubiales, G.; de los Santos, J. M. Tetrahedron 2007, 63, 523.

(b) Fresneda, P. M.; Molina, P. Synlett 2004, 1.

[50] (a) Largeron, M.; Fleury, M.-B. Angew. Chem., Int. Ed. 2012, 51, 5409.

(b) Lang, X.; Ji, H.; Chen, C.; Ma, W.; Zhao, J. Angew. Chem., Int. Ed. 2011, 50, 3934.

(c) Jiang, G.; Chen, J.; Huang, J.-S.; Che, C.-M. Org. Lett. 2009, 11,4568 .

(d) Samec, J. S. M.; Éll, A. H.; Bäckvall, J.-E. Chem. Eur. J. 2005, $11,2327$.

[51] Schiff, H. Annals 1864, 131, 118.

[52] Medvedeva, A. S.; Mareev, A. V.; Borisova, A. I.; Afonin, A. V. ARKIVOC 2003, 13, 157.

[53] Yusubov, M. S.; Chi, K.-W.; Park, J. Y.; Karimovc, R.; Zhdankinc, V. V. Tetrahedron Lett. 2006, 47, 6305.

[54] (a) Gnanaprakasam, B.; Zhang, J.; Milstein, D. Angew. Chem., Int. Ed. 2010, 49, 1468.

(b) Maggi, A.; Madsen, R. Organometallics 2012, 31, 451.

(c) Esteruelas, M. A.; Honczek, N.; Oliván, M.; Onate, E.; Valencia, M. Organometallics 2011, 30, 2468.

(d) Cano, R.; Ramón, D. J.; Yus, M. J. Org. Chem. 2011, 76, 5547.

(e) Shiraishi, Y.; Ikeda, M.; Tsukamoto, D.; Tanaka, S.; Hiraia, T. Chem. Commun. 2011, 47, 4811.

[55] Sun, H.; Su, F.-Z.; Ni, J.; Cao, Y.; He, H.-Y.; Fan, K.-N. Angew. Chem., Int. Ed. 2009, 48, 4390.

[56] Kim, J. W.; He, J.; Yamaguchi, K.; Mizuno, N. Chem. Lett. 2009, $38,920$.

[57] Kegnaes, S.; Mielby, J.; Mentzel, U. V.; Christensen, C. H.; Riisager, A. Green Chem. 2010, 12, 1437.

[58] He, W.; Wang, L.; Sun, C.; Wu, K.; He, S.; Chen, J.; Wu, P.; Yu, Z. Chem. Eur. J. 2011, 17, 13308.

[59] Kang, Q.; Zhang, Y. Green Chem. 2012, 14, 1016.

[60] Sithambaram, S.; Kumar R.; Son, Y.-C.; Suib, S. L. J. Catal. 2008, 253, 269.

[61] Kwon, M. S.; Kim, N.; Seo, S. H.; Park, I. S.; Cheedrala, R. K.; Park, J. Angew. Chem., Int. Ed. 2005, 44, 6913.

[62] Kim, S.; Bae, S. W.; Lee, J. S.; Park, J. Tetrahedron 2009, 65, 1461.

[63] Xu, Q.; Tian, H.; Jin, L. CN 102775288, 2012 [Chem. Abstr. 2012, 158, 11329].

(Cheng, F.) 